\title{
Wind Conditions in Idealized Building Clusters: Macroscopic Simulations Using a Porous Turbulence Model
}

\author{
Jian Hang • Yuguo Li
}

Received: 18 March 2009 / Accepted: 19 March 2010 / Published online: 17 April 2010

(C) The Author(s) 2010. This article is published with open access at Springerlink.com

\begin{abstract}
Simulating turbulent flows in a city of many thousands of buildings using general high-resolution microscopic simulations requires a grid number that is beyond present computer resources. We thus regard a city as porous media and divide the whole hybrid domain into a porous city region and a clear fluid region, which are represented by a macroscopic $k-\varepsilon$ model. Some microscopic information is neglected by the volume-averaging technique in the porous city to reduce the calculation load. A single domain approach is used to account for the interface conditions. We investigated the turbulent airflow through aligned cube arrays (with 7, 14 or 21 rows). The building height $H$, the street width $W$, and the building width $B$ are the same $(0.15 \mathrm{~m})$, and the fraction of the volume occupied by fluid (i.e. the porosity) is 0.75 ; the approaching flow is parallel to the main streets. There are both microscopic and macroscopic simulations, with microscopic simulations being well validated by experimental data. We analysed microscopic wind conditions and the ventilation capacity in such cube arrays, and then calculated macroscopic time-averaged properties to provide a comparison for macroscopic simulations. We found that the macroscopic $k-\varepsilon$ turbulence model predicted the macroscopic flow reduction through porous cube clusters relatively well, but under-predicted the macroscopic turbulent kinetic energy (TKE) near the windward edge of the porous region. For a sufficiently long porous cube array, macroscopic flow quantities maintain constant conditions in a fully developed region.
\end{abstract}

Keywords Building array $\cdot$ Macroscopic simulation $\cdot$ Porosity $\cdot$ Porous media · Porous turbulence model

\footnotetext{
J. Hang · Y. Li (ه)

Department of Mechanical Engineering, The University of Hong Kong, Haking Wong Building, Pokfulam Road, Hong Kong SAR, China

e-mail: liyg@hku.hk

J. Hang

e-mail: hangjian@hku.hk
} 


\section{Introduction}

Modern compact cities generally consist of agglomerations of buildings and other solid obstacles, with small voids between neighbouring buildings and large voids such as street networks. Though the urban canopy layer provides residents with shelter, protection and convenience, it also disturbs the turbulent flow in the atmospheric boundary layer and causes a blockage to the approaching flow. In crowded cities, concentrated human activities may produce a large amount of airborne contaminants (Fenger 1999). A study of wind conditions in the urban canopy layer of a city aids the understanding of urban airflow in redistributing and/or removing airborne pollutants. The question is how to quantify the airflow below the canopy layer. In analogy to the ventilation of buildings (Etheridge and Sandberg 1996), the concept of ventilation flow rates and the air exchange rate $\mathrm{ACH}$, i.e. the air change rate per hour) may be introduced. The city ventilation flow rates refer to the amount of external air entering and flowing through the urban canopy layer. The ACH for a space in the urban canopy layer represents the relative amount of air exchange between the space and the external air surrounding it, which can be calculated via the volumetric ventilation flow rates and the volume of the space.

Computational fluid dynamics (CFD) simulations have been used to predict urban wind conditions. Previous studies may be divided into two types, i.e. the mesoscale type (up to 10-200 km horizontal scale) and the microscale type (up to $100 \mathrm{~m}$ to $2 \mathrm{~km}$ horizontal scale). The former type (Liu et al. 2001; Ding et al. 2004; Tong et al. 2005) usually considers a city as an enhanced surface roughness, and emphasizes the effects of geographic and meteorological conditions as well as regional pollutant transport on wind conditions and urban pollution on a larger scale, without any flow information below the urban canopy layer on the building or street scale. The latter type studies turbulent airflows and pollutant dispersion on the microscale, i.e. around isolated buildings ( $\mathrm{Li}$ and Stathopoulos 1997), in two-dimensional (2D) street canyons (Oke 1988; Sini et al. 1996; Baik and Kim 2002; Liu et al. 2005; Xie et al. 2006; Li et al. 2009), in groups of building arrays (Hanna et al. 2002; Chang and Meroney 2003; Britter and Hanna 2003; Belcher 2005; Martilli and Santiago 2007; Santiago et al. 2007, 2008) using large-eddy simulation (LES) or microscopic simulations with Reynoldsaveraged Navier-Stokes (RANS) turbulence models or wind-tunnel experiments. Cheng et al. (2003) investigated the turbulent flow over a matrix of cubes with a LES model and a RANS standard $k-\varepsilon$ model. They reported that the LES model produces the turbulence structure more precisely than the RANS standard $k-\varepsilon$ model, but with a calculation load nearly 100 times greater than the RANS turbulence model.

We aim to study wind conditions in a city with a scale of up to $2 \mathrm{~km}$ or more than $10 \mathrm{~km}$, in which thousands of buildings are included, and also quantify wind conditions on a street scale. We treat a city with agglomerations of building obstacles as porous media made up of solid buildings with street voids between them. The airflow in a porous city region is solved by macroscopic simulations with a porous turbulence model, in which some microscopic information is neglected by the volume-averaging technique and the turbulent airflow between individual obstacle elements is unresolved, while the effects of the building cluster on the urban airflow are modelled as sink or source terms for momentum and turbulence in the transport equations.

Turbulence and transport flow in porous media have been found in many industries and engineering applications, including chemical reactors or heat exchangers, plant canopies or soil mechanics, etc. The flow properties in porous models depend on the interaction of the overlying clear-fluid region with the porous region, the porosity and the permeability of porous media. General porous models in previous studies were derived from the 
volume-averaging technique on a representative elementary volume (REV) and based on Darcy's Law or its extension. These models focused on the global flow characteristics in the REV and neglected detailed flow information. All the volume-averaging techniques on microscopic equations generate several additional terms to model the viscous and drag forces in porous media together providing closure problems for these additional terms (Antohe and Lage 1997; Nakayama and Kuwahara 1999; Getachew et al. 2000; Pedras and de Lemos 2000; Lien et al. 2005; Chandesris et al. 2006; Kuwahara et al. 2006). Flow through a packed city can be assumed to be an incompressible turbulent flow in a rigid isotropic and fixed porous matrix with high Reynolds number. The experientially based BrinkmanForchheimer-extended Darcy (BFD) flow model is widely used, possibly because of its similarity to the Navier-Stokes equations. Regarding this model, Antohe and Lage (1997) mathematically developed a two-equation macroscopic turbulence model for high Reynolds number incompressible flow within fluid-saturated and rigid porous media, in which the closure coefficients are the same as those in the standard $k-\varepsilon$ turbulence model. In their derivation, while modelling the form drag, only the Forchheimer terms that are linear in the fluctuating velocities were retained. In their conclusion, for porous media of small permeability, the effect of the solid matrix is to damp the turbulence, while in the case of large permeability, it can enhance or damp turbulence. Getachew et al. (2000) pointed out that neglecting the higher-order part may lose some significant effects of the Forchheimer term because most of the statistical properties of turbulence are in the second-order correlation terms. So they added the second-order correlation term into the Forchheimer term. The additional higher-order terms produce additional correlation coefficients. The model derived by Antohe and Lage (1997) and modified by Getachew et al. (2000) may be considered as the most comprehensive turbulence models used for flows in porous media and the most suitable one for this research. Because several closure coefficients in Getachew's model are still unknown and should be decided by numerical experiments using LES or direct numerical simulation (DNS), we suggest using the model of Antohe and Lage (1997) for simulations in future. Prakash et al. (2001a,b) studied the way that the porous features affect low Reynolds number flow in the overlying fluid layer using the porous turbulence model developed by Antohe and Lage (1997) and LDV visualization measurements, in which the Reynolds number, the effect of permeability, the height of the fluid layer and the thickness of the porous media were estimated. They reported that the permeability of porous foam and the height of the overlying fluid layer strongly influence the flow pattern in the overlying fluid region. They also demonstrated the significant role of the interaction between the porous region and the overlying clear-fluid region. There are few studies that use the porous turbulence model developed by Antohe and Lage (1997) for a porous urban canopy flow.

Brown et al. (2001) performed a detailed wind-tunnel experiment of a group of low-rise aligned seven-row cube building arrays $(H=W=B=0.15 \mathrm{~m}$, the fraction of volume occupied by air voids i.e. porosity $\phi=0.75$ ), using a pulsed-wire anemometer (PWA). Lien and Yee (2004); Lien et al. (2005) carried out a detailed microscopic simulation of airflow in similar cube arrays and then performed macroscopic simulations using a modified $k-\varepsilon$ turbulence model to study macroscopic airflow through and over porous media with cubic arrays. In this research, the higher-order approximations of the source/sink terms in the $k-\varepsilon$ equations did not offer any predictive advantage. So we use the porous turbulence models developed by Antohe and Lage (1997) to study the flow reduction through idealized porous building clusters. Santiago et al. (2007) performed a detailed microscopic CFD simulation based on a RANS standard $k-\varepsilon$ turbulence model and analysed the flow structure in such a cube array with detailed validation by wind-tunnel data. Then Martilli and Santiago (2007) and Santiago et al. (2008) analysed the drag coefficient and spatially-averaged properties in this cube array. 
It may be mentioned that, following the model of Finnigan (2000) for flows in vegetation canopies, Belcher et al. (2003) and Coceal and Belcher (2005) developed theoretical models to study the spatially-averaged mean flow and macroscopic velocity reduction through sparse porous cube arrays (porosity $=0.89$ ) where the gap between obstacles is large. However, these theoretical models are invalid for the low-rise medium (porosity $=0.75$ ) cube array $(H / W=1)$ because the skimming flow regime exists in such obstacle arrays.

\section{Model Descriptions and Methodology}

\subsection{Microscopic and Macroscopic Equations}

The urban airflow is a turbulent flow through, around and over groups of porous building clusters with different porosities and building heights. The whole domain consists of a porous city region and a clear airflow region with a macroscopic interface. We first study porous cube arrays with a single porosity and uniform building height.

There are two kinds of average operators on the Navier-Stokes equations, the timeaverage operator and local volume-average operator. With the time-average operator, the general fluid property $\psi$ can be divided into the time average $\bar{\psi}$ and the time fluctuating component $\psi^{\prime}$,

$$
\bar{\psi}=\frac{1}{\Delta t} \int_{t}^{t+\Delta t} \psi d t,
$$

where $\psi=\bar{\psi}+\psi^{\prime}$. With the volume-average operator, the property $\psi$ can be divided into the intrinsic average $\langle\psi\rangle^{f}$ and its spatial variation $\psi^{i}$ in porous media,

$$
\langle\psi\rangle^{f}=\frac{1}{\Delta V_{f}} \int_{\Delta V_{f}} \psi d V
$$

where $\psi=\langle\psi\rangle^{f}+\psi^{i}$. In addition, the volume average $\langle\psi\rangle^{v}$ and the intrinsic average $\langle\psi\rangle^{f}$ are related by the porosity $\phi$ in porous media,

$$
\begin{aligned}
\langle\psi\rangle^{v} & =\frac{1}{\Delta V} \int_{\Delta V_{f}} \psi d V, \\
\phi & =\frac{\Delta V_{f}}{\Delta V},
\end{aligned}
$$

where $\langle\psi\rangle^{v}=\phi\langle\psi\rangle^{f}, \Delta V_{f}$ is the fluid volume in a special REV $\Delta V$ and $\phi$ is the fraction of fluid volume in porous media, i.e. the porosity.

The well-known RANS models, including $k-\varepsilon$ turbulence models and the Reynolds stress model (RSM), are the most commonly used to close the time-averaged Navier-Stokes equations. Here we use the standard $k-\varepsilon$ turbulence model to estimate the mean flow and turbulence characteristics for stationary, incompressible, and isothermal flow conditions in the clear fluid region (outside of the porous city region) in macroscopic simulations, or in the whole flow field in high-resolution microscopic simulations when there are not too many buildings and the calculation load is not too great. The microscopic time-averaged governing equations include the continuity equation, 


$$
\frac{\partial\left(\rho \overline{u_{i}}\right)}{\partial x_{i}}=0
$$

the momentum conservation equation,

$$
\frac{\partial\left(\rho \overline{u_{i} u_{j}}\right)}{\partial x_{j}}=\frac{\partial}{\partial x_{j}}\left[\left(\mu+\mu_{t}\right) \frac{\partial \overline{u_{i}}}{\partial x_{j}}\right]-\left(\frac{\partial p}{\partial x_{i}}+\frac{2}{3} \rho \frac{\partial k}{\partial x_{i}}\right),
$$

and two transport equations for turbulent kinetic energy (TKE) and its dissipation rate,

$$
\begin{aligned}
& \frac{\partial\left(\rho \overline{u_{j}} k\right)}{\partial x_{j}}=\frac{\partial}{\partial x_{j}}\left[\left(\mu+\frac{\mu_{t}}{\sigma_{k}}\right) \frac{\partial k}{\partial x_{j}}\right]+G_{k}-\rho \varepsilon, \\
& \frac{\partial\left(\rho \overline{u_{j}} \varepsilon\right)}{\partial x_{j}}=\frac{\partial}{\partial x_{j}}\left[\left(\mu+\frac{\mu_{t}}{\sigma_{\varepsilon}}\right) \frac{\partial \varepsilon}{\partial x_{j}}\right]+c_{\varepsilon 1} \frac{\varepsilon}{k} G_{k}-\rho c_{\varepsilon 2} \frac{\varepsilon^{2}}{k},
\end{aligned}
$$

where the closure coefficients $\left(C_{\varepsilon 1}, C_{\varepsilon 2}, C_{\mu}, \sigma_{k}, \sigma_{\varepsilon}\right)=(1.44,1.92,0.09,1.0,1.3)$. In Eqs. 3-6, $\mu_{t}=\rho C_{\mu} k^{2} / \varepsilon$ is the turbulent viscosity, $\mu$ is the viscosity and $G_{k}$ is the turbulence kinetic energy production $\left(=\mu_{t} \frac{\partial \overline{u_{i}}}{\partial x_{j}}\left(\frac{\partial \overline{u_{i}}}{\partial x_{j}}+\frac{\partial \overline{u_{j}}}{\partial x_{i}}\right)\right)$. All the variables $\left(\overline{u_{i}}, p, k, \varepsilon\right)$ here are microscopic time-averaged quantities (i.e. the mean velocity components, pressure, turbulence kinetic energy and its dissipation rate).

The macroscopic time-averaged quantities (i.e. the volume average of time-averaged variables) for flows through porous media can be acquired by volume averaging the corresponding microscopic time-averaged quantities over a volume of REV $(\Delta V)$.

$$
\begin{aligned}
\langle\bar{\psi}\rangle^{v} & =\frac{1}{\Delta V} \int_{\Delta V_{f}} \bar{\psi} d V, \\
\langle\bar{\psi}\rangle^{f} & =\frac{1}{\Delta V_{f}} \int_{\Delta V_{f}} \bar{\psi} d V, \\
\langle\bar{\psi}\rangle^{v} & =\phi\langle\bar{\psi}\rangle^{f},
\end{aligned}
$$

where $\langle\bar{\psi}\rangle^{v}$ and $\langle\bar{\psi}\rangle^{f}$ are the volumetric and intrinsic averages of a time-averaged quantity $\bar{\psi}$ that are related by the porosity $\phi$. To simplify the equations, we write macroscopic timeaveraged variables $\langle\bar{\psi}\rangle^{f}$ (i.e. the spatial intrinsic averages of microscopic time-averaged variables) into $\psi^{f}$ in the following governing equations.

There are two methods of deriving the macroscopic transport equations for airflow in porous media depending on the order of application of the two operators. Pedras and de Lemos (2001) found that the final form of the momentum transport equations from both methods is the same. However, those in turbulence models are different. Antohe and Lage (1997) derived a macroscopic turbulence $k-\varepsilon$ model for incompressible flows in porous media by time averaging the general equations for high Reynolds number incompressible flow within fluid-saturated and rigid porous media. The transport equations can be written in the following conservative form:

$$
\begin{gathered}
\frac{\partial\left(\rho \phi u_{i}^{f}\right)}{\partial x_{i}}=0 \\
\frac{\partial\left(\rho \phi u_{i}^{f} u_{j}^{f}\right)}{\partial x_{j}}=\frac{\partial}{\partial x_{j}}\left[\left(\mu J+\mu_{t}\right) \frac{\partial \phi u_{i}^{f}}{\partial x_{j}}\right]-\left(\frac{\partial \phi p^{f}}{\partial x_{i}}+\frac{2}{3} \rho \frac{\partial \phi k^{f}}{\partial x_{i}}\right)
\end{gathered}
$$




$$
\begin{aligned}
& \overbrace{-\phi \frac{\mu}{K} \phi u_{i}^{f}}^{\text {udarcy }} \overbrace{-\phi \frac{\rho C_{F}}{\sqrt{K}} \phi Q^{f} \phi u_{i}^{f}}^{\text {uForch }}, \\
& \frac{\partial\left(\rho \phi u_{j}^{f} k^{f}\right)}{\partial x_{j}}=\frac{\partial}{\partial x_{j}}\left[\left(\mu J+\frac{\mu_{t}}{\sigma_{k}}\right) \frac{\partial \phi k^{f}}{\partial x_{j}}\right]-\rho \phi \varepsilon f \overbrace{+\phi G_{k}}^{\text {TKEgen }} \overbrace{-2 \phi \frac{\mu}{K} \phi k^{f}}^{\text {TKEdarcy }} \\
& \overbrace{-\phi^{2} \frac{C_{F}}{\sqrt{K}} \frac{8}{3} \rho Q^{f} \phi k^{f}}^{\text {TKEForch }}+\overbrace{2 \phi \phi^{2} \frac{C_{F}}{\sqrt{K}} F_{k}}^{\text {TKE_Fk }} \\
& \frac{\partial\left(\rho \phi u_{j}^{f} \varepsilon^{f}\right)}{\partial x_{j}}=\frac{\partial}{\partial x_{j}}\left[\left(\mu J+\frac{\mu_{t}}{\sigma_{\varepsilon}}\right) \frac{\partial \phi \varepsilon^{f}}{\partial x_{j}}\right]+c_{\varepsilon 1} \phi \frac{\varepsilon^{f}}{k^{f}} G_{k}-J \rho c_{\varepsilon 2} \phi \frac{\varepsilon^{f}}{k^{f}} \varepsilon^{f} \\
& \overbrace{-2 \phi \frac{\mu}{K} \phi \varepsilon^{f}}^{\text {EDdarcy }} \overbrace{-\frac{8}{3} \phi^{3} \frac{C_{F}}{\sqrt{K}} \rho Q^{f} \varepsilon^{f}}^{\text {EDForch1 }} \overbrace{-\frac{8 \mu}{3} \phi^{3} \frac{C_{F}}{\sqrt{K}} \frac{\partial k^{f}}{\partial x_{r}} \frac{\partial Q^{f}}{\partial x_{r}}}^{\text {EDForch2 }} \\
& \text { EDForch3 } \\
& +2 \overbrace{}^{2} \phi \frac{C_{F}}{\sqrt{K}}\left[\mu v_{t} \frac{\partial}{\partial x_{r}}\left(\frac{u_{j}^{f} u_{i}^{f}}{Q^{f}}\right) \frac{\partial^{2} u_{i}^{f}}{\partial x_{r} \partial x_{j}}+2 \mu v_{t} \frac{u_{j}^{f} u_{i}^{f}}{Q^{f}} \frac{\partial^{2}}{\partial x_{r}^{2}}\left(\frac{\partial u_{i}^{f}}{\partial x_{j}}\right)\right], \\
& Q^{f}=\sqrt{u_{i}^{f} u_{i}^{f}}, \\
& \mu_{t}=C_{\mu} \rho \frac{k^{f 2}}{\varepsilon^{f}}, \\
& G_{k}=\mu_{t} \frac{\partial u_{i}^{f}}{\partial x_{j}}\left(\frac{\partial u_{i}^{f}}{\partial x_{j}}+\frac{\partial u_{j}^{f}}{\partial x_{i}}\right), \\
& F_{k}=\mu_{t} \frac{u_{j}^{f} u_{i}^{f}}{Q^{f}} \frac{\partial u_{j}^{f}}{\partial x_{i}},
\end{aligned}
$$

where $\rho$ is the fluid density, $u_{i}^{f}, p^{f}, k^{f}, \varepsilon^{f}$ are intrinsic averages of time-averaged variables (the velocity components, pressure, TKE and its dissipation rate), $\mu$ and $\mu_{t}$ are the dynamic and turbulent viscosities, respectively, $J=\tilde{\mu} / \mu$ is the viscosity ratio that can be assumed to be equal to one for most applications in porous media (Antohe and Lage 1997), $K$ is the permeability, and $C_{F}$ is the Forchheimer coefficient. We used the same constant coefficients as those in Eqs. 5 and $6 .^{1}$

1 There are some errors in the derivation for the transport equation of $\varepsilon^{f}$ [see Eqs. 28-31 in Antohe and Lage (1997)]. For example, in Eq. 28 of Antohe and Lage (1997), the derivation $\frac{1}{2} \frac{\partial Q}{\partial x_{r}} \frac{\partial}{\partial x_{r}}\left(\overline{\left.u_{i}^{f^{\prime}} u_{i}^{f^{\prime}}\right)}=\right.$ $\frac{1}{2} \frac{\partial Q}{\partial x_{r}} \frac{\partial k^{f}}{\partial x_{r}}$ is wrong and should be $\frac{1}{2} \frac{\partial Q}{\partial x_{r}} \frac{\partial}{\partial x_{r}}\left(\overline{u_{i}^{f^{\prime}} u_{i}^{f^{\prime}}}\right)=\frac{\partial Q}{\partial x_{r}} \frac{\partial k^{f}}{\partial x_{r}}$. So EDForch2 and EDForch3 in the present equations are different from those in the original equations in Antohe and Lage (1997) (i.e. EDForch2 
In the right-hand side of Eq. 9, there are the pressure gradient term $\left(-\partial\left(\varphi p^{f}\right) / \partial x_{i}\right)$, the viscous diffusion term $\left(\frac{\partial}{\partial x_{j}}\left[\mu J \frac{\partial \varphi u_{i}^{f}}{\partial x_{j}}\right]\right)$ and the eddy diffusion term $\left(\frac{\partial}{\partial x_{j}}\left[\mu_{t} \frac{\partial \varphi u_{i}^{f}}{\partial x_{j}}\right]\right.$ $\left.-\frac{2}{3} \rho \frac{\partial \varphi k^{f}}{\partial x_{i}}\right)$, which is from the Reynolds stresses $\left(-\mu_{t}\left(\frac{\partial \varphi u_{i}^{f}}{\partial x_{j}}+\frac{\partial \varphi u_{j}^{f}}{\partial x_{i}}\right)+\frac{2}{3} \rho \varphi k \delta_{i j}\right)$. The Darcy term $\left(-\phi \frac{\mu}{K} \phi u_{i}^{f}\right.$, i.e. udarcy term $)$ and the Forchheimer term $\left(-\phi \frac{\rho C_{F}}{\sqrt{K}} \phi Q^{f} \phi u_{i}^{f}\right.$, i.e. uForch term) are used to model microscopic viscous drag generated by the fluid itself and microscopic form drag produced by solid particles in porous media (or buildings in a porous city), which act as sink terms for the momentum. Similarly, in the right-hand side of Eq. 10, the Darcy term (i.e. TKEdarcy term) and the first part (i.e. TKEForch term) of the Forchheimer terms are always negative, which implies that they enhance the reduction of TKE in porous media. However, the second part (i.e. TKE_Fk term) of the Forchheimer terms for TKE may act as a sink or source term for TKE because it may be either negative or positive in the porous media depending on the velocity components and their derivatives. In Eq. 11, the Darcy term $\left(-2 \mu \phi^{2} \varepsilon^{f} / K\right.$, i.e. the EDdarcy term) is always negative, which results in a decrease in the dissipation rate of TKE, i.e. weakening the depletion of TKE. The Forchheimer terms of Eq. 11 include three parts, in which the first part $\left(-\frac{8}{3} \phi^{3} C_{F} \rho Q^{f} \varepsilon^{f} / \sqrt{K}\right.$, i.e. EDForch1 term) works in the same way as the Darcy term (i.e. EDdarcy). However, the other two parts (i.e. EDForch2 and EDForch3 terms) may be positive or negative, and this is decided by the fluid viscosity $\mu$, the velocity components and their derivatives.

\subsection{Interface Conditions}

The urban airflow is a kind of turbulent flow through and over a porous city with finite porosity and permeability. The whole domain consists of a porous city region and a clear fluid region with a macroscopic interface. For turbulent flows through, around and over a porous city, we use Eqs. 3-6 to solve the flow field in the clear fluid region, and make use of Eqs. 8 to $12 \mathrm{a}-12 \mathrm{~d}$ to model the macroscopic time-averaged variables in the porous city region. The interaction at the interface between the urban canopy layer (the porous city region) and the atmospheric boundary layer (the clear fluid region) plays a significant role in the flow in the porous urban canopy layer. So the mathematical treatments at the interface between the two regions are very important. In previous general studies, there are different issues about interface conditions for the transport equations of mass (fluid, pollutant, heat), momentum, and turbulence ( $k$ and $\varepsilon$ ). One issue involves utilization of the interface shear-stress jump condition between the two regions, in which the definition of the interface condition only depends on an empirically decided constant. Silva and de Lemos (2003) and de Lemos (2005) investigated both laminar and turbulent flow over porous media with this kind of jump interface condition, and it was found that a small change in the value of the stress jump parameters results in a very different structure for the turbulence flow field. Another significant issue is the classical continuity boundary conditions, i.e. no jump condition at the interfaces for all

Footnote 1 continued

$=-\frac{5 \mu}{3} \phi^{3} \frac{C_{F}}{\sqrt{K}} \frac{\partial k^{f}}{\partial x_{r}} \frac{\partial Q^{f}}{\partial x_{r}}$ EDForch3 $\left.=2 \phi^{2} \phi \frac{C_{F}}{\sqrt{K}}\left[2 \mu v_{t} \frac{\partial}{\partial x_{r}}\left(\frac{u_{j}^{f} u_{i}^{f}}{Q^{f}}\right) \frac{\partial^{2} u_{i}^{f}}{\partial x_{r} \partial x_{j}}+\mu v_{t} \frac{u_{j}^{f} u_{i}^{f}}{Q^{f}} \frac{\partial^{3} u_{i}^{f}}{\partial x_{j} \partial x_{r}^{2}}\right]\right)$. We find that some different constants are used in the terms of EDForch2 and EDForch3 between the present equations and Antohe and Lage's original model. The effect of such differences will be checked and discussed using numerical simulation results. 
the variables. Choi and Waller (1997) developed the classical continuity boundary conditions to deal with the transportation problem at the interface in a laminar regime. Chan et al. (2007) extended this condition to the turbulent regime, and it was found that the penetration extent of turbulence was Darcy-number dependent and porosity dependent. We used a third approach, i.e. a single domain approach (Neale and Nader 1974; Prakash et al. 2001a). In this approach, if the porosity $\phi$ is 1 , the permeability $K$ has a finite value and the Forchheimer coefficient $C_{F}$ is zero. Equations 8 to $12 \mathrm{a}-12 \mathrm{~d}$ for porous regions become the same as those in the clear fluid region (Eqs. 3-6). So we treated the two regions as a single domain and used the same equations (Eqs. 8 to 12a-12d) for both regions, by using different parameters (the porosity $\phi$, the permeability $K$ and the Forchheimer coefficient $C_{F}$ ).

We developed a 'Ventair' code using Fortran language to carry out both microscopic and macroscopic simulations. For macroscopic simulations using the porous turbulence model, we defined an interface between the porous city region and the clear fluid region. We used distributions of $\phi(x, y, z), K(x, y, z)$ and $C_{F}(x, y, z)$ to define parameters in the two regions.

\subsection{Experimental Model Description}

As shown in Fig. 1a, Brown et al. (2001) performed detailed wind-tunnel measurements on a group of low-rise aligned seven-row (in the streamwise $(x)$ direction) and 11-column (in the lateral ( $y$ ) direction) cube building array $(H=W=B=0.15 \mathrm{~m})$. The working test section of the wind tunnel is $18.3 \mathrm{~m}$ long, $3.7 \mathrm{~m}$ wide and $2.1 \mathrm{~m}$ high, in which a neutrally stratified atmospheric boundary layer with a depth of $1.8 \mathrm{~m}$ and a friction velocity $u_{*}=0.24 \mathrm{~m} \mathrm{~s}^{-1}$ was generated in the upstream flow of the cube array using a roughness length of $0.001 \mathrm{~m}$. The mean streamwise velocity in the upstream free flow was described by a power-law profile $\left(U_{0}(z)=U_{H}(z / H)^{0.16}\right)$, and $U_{H}=3.0 \mathrm{~m} \mathrm{~s}^{-1}$ is the reference velocity of the upstream free flow at $z=H$. The Reynolds number $\left(R_{e H}=\rho U_{H} H / \mu \approx 30,000\right)$ is sufficiently high to ensure Reynolds-number independence (Meroney et al. 1996).

Vertical profiles of the three velocity components and TKE at many points of the vertical symmetric plane (i.e. in the plane of $y / H=0$ ) were presented in Brown et al. (2001). As shown in Fig. 1a, we define $x / H=0$ as the location of the windward edge of the first row of cubes. The seven buildings are named as buildings No. 1-7. The canyons behind buildings No. 1-6 are named as canyons No. 1-6. Figure $1 \mathrm{~b}$ shows the detailed information of these points in the plane of $y / H=0$ : points $\mathrm{C} 1, \mathrm{C} 2, \mathrm{C} 3, \mathrm{C} 4, \mathrm{C} 5$ and $\mathrm{C} 6$ are the centre points inside canyons Nos. 1, 2, 3, 4, 5 and 6, for which the locations are $x / H=1.5,3.5,5.5,7.5,9.5$ and 11.5. In addition, Points $\mathrm{C} 0$ and $\mathrm{C} 7$ are at $x / H=-0.5$ (i.e. the windward side of building No. 1) and 16.5 (i.e. the leeward side of building No. 7), respectively. Points B1, B2, B6 and B7 are the centre points of building rooftops for buildings Nos. 1, 2, 6 and 7.

\subsection{Numerical Model Descriptions}

To reduce the calculation time in the microscopic simulation, we only considered the middle column of the cube array and only used half of this column that is surrounded by dots in Fig. 1a, so the computational domain is only $H$ wide in the lateral (i.e. $y$ ) direction. In addition, the computational domain is $8 H$ high in the vertical (i.e. $z$ ) direction. In the streamwise direction (i.e. $x$ ), it is $6.6 \mathrm{H}$ long from the upstream domain inlet to the windward edge of the first row of cubes, and $20.3 \mathrm{H}$ long from the leeward edge of the final building to the downstream domain outlet. To study the dependence of the flow solution on grid resolution, we first used two types of grids. For a coarse grid $(235 \times 23 \times 47)$, uniform grids in the $x$ and 
(a)

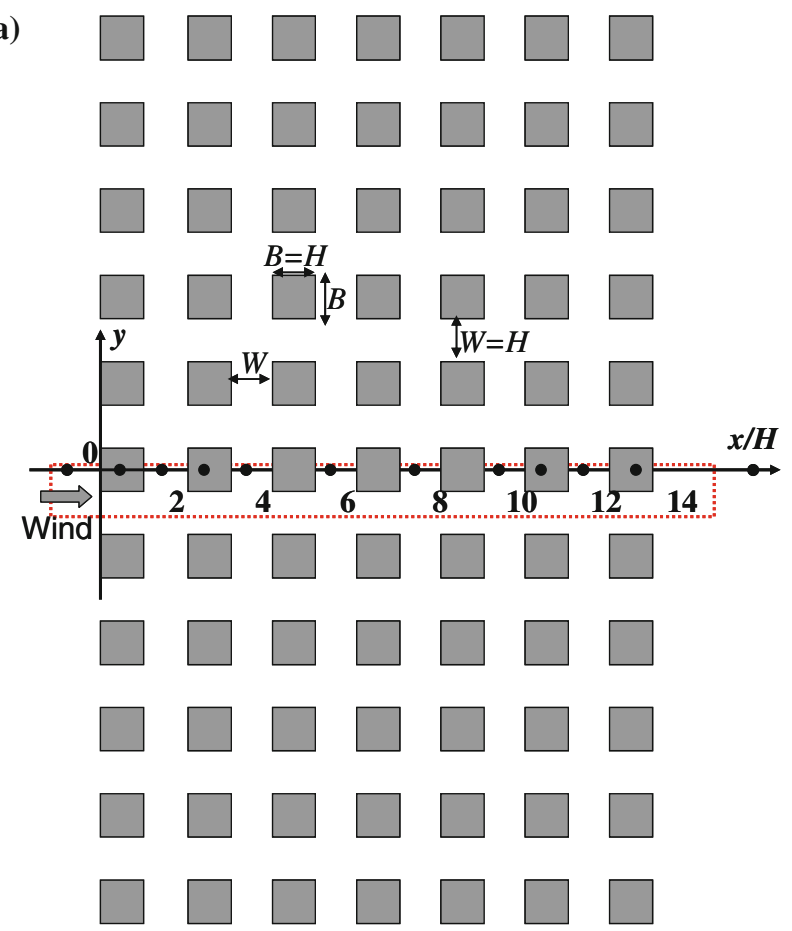

(b)

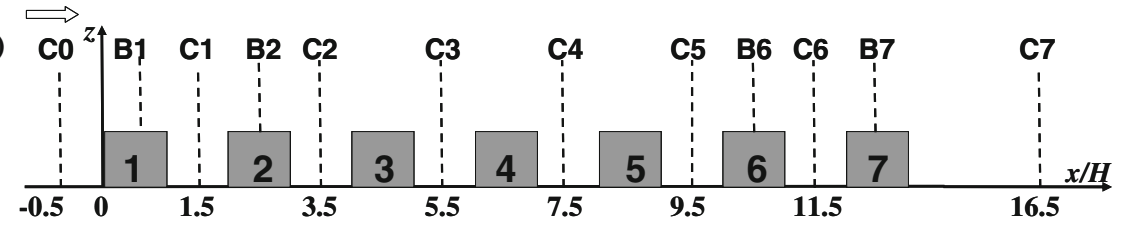

(c)
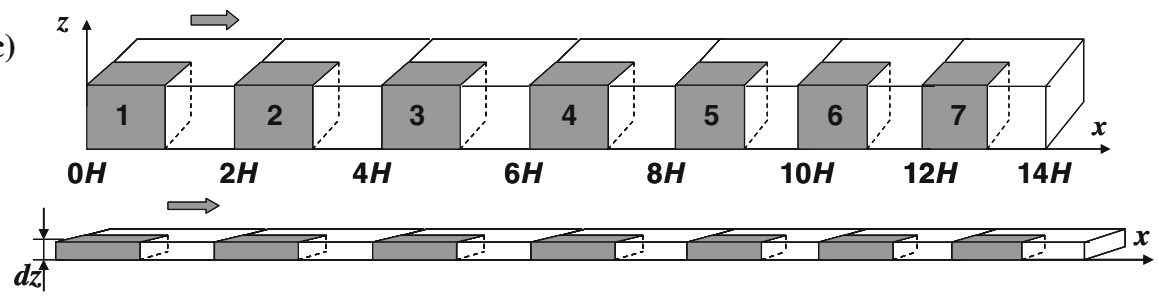

(d)

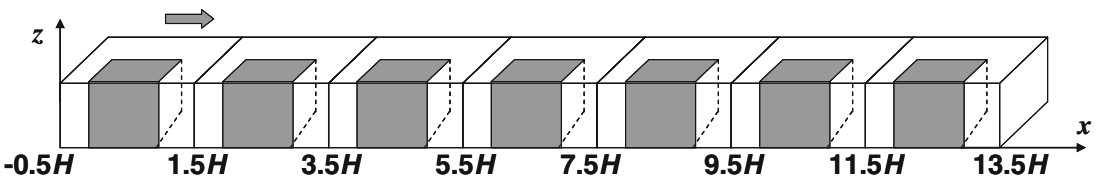

Fig. 1 Model description of wind-tunnel experiments and vertical profiles at several points in Brown et al. (2001). a $x-y$ view and $\mathbf{b} x-z$ view in the plane of $y / H=0$. Definitions of an aligned seven-row cube array $(W=H)$ into seven REV units with two methods: $\mathbf{c}$ Method 1 (seven REV units and seven thin horizontal slabs) and d Method 2 (seven REV units) 

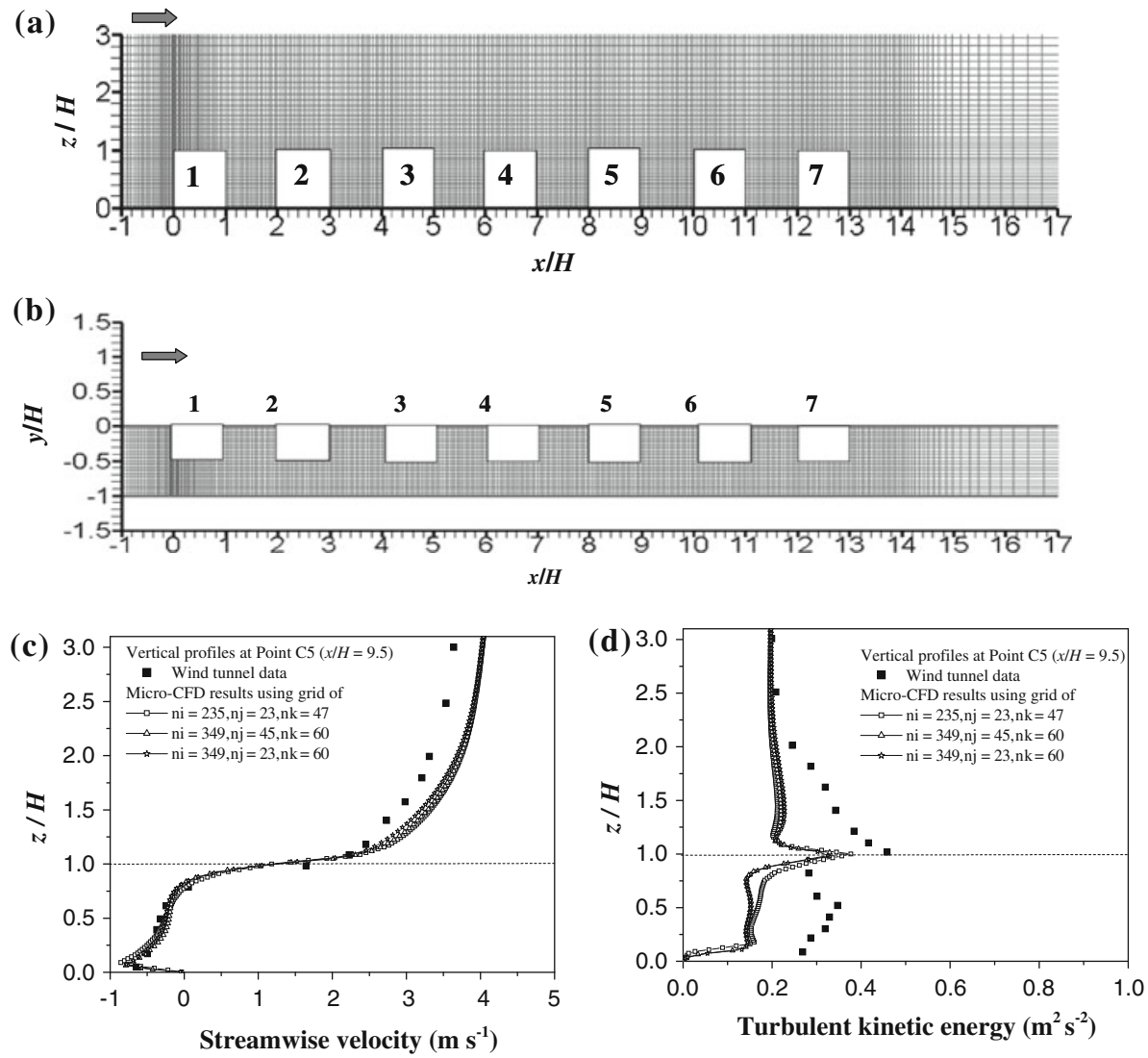

Fig. 2 Grid generation of the medium grid $(349 \times 23 \times 60)$ within and around a seven-row cube array, a in the plane $y / H=0$, and $\mathbf{b}$ in the plane $z / H=0$. Grid-sensitivity analysis using vertical profiles at Point C5 in the seven-row cube array $\mathbf{c}$ streamwise velocity and $\mathbf{d}$ TKE

$z$ directions inside the canyons were used with a grid size of $0.0125 \mathrm{~m}$ (i.e. 12 points), and the uniform grid size in the $y$ direction was $0.0068 \mathrm{~m}$. For a fine grid $(349 \times 45 \times 60)$, uniform grids in the $x$ and $z$ directions inside the canyons were used with a grid size of $0.0083 \mathrm{~m}$ (i.e. 18 points) and the uniform grid size in the $y$ direction was $0.0034 \mathrm{~m}$. The grid size in the streamwise direction $(x)$ increases from the first row of cubes to the upstream domain inlet and from the last row of cubes to the downstream domain outlet, with a ratio of 1.05 and 1.1, respectively. The grid size in the vertical direction (i.e. $z$ ) above the cube buildings also increases with a ratio of 1.05 .

Figure $2 \mathrm{c}$ and $\mathrm{d}$ shows vertical profiles of the mean streamwise velocity and TKE at Point C5 $(x / H=9.5)$. The difference in the mean streamwise velocity between the coarse and fine grids is small but the profiles of TKE with the coarse and fine grids are a little different. So we also used a medium grid $(349 \times 23 \times 60)$, i.e. the same grid as the fine grid in the streamwise direction and the vertical direction, and the same grid as the coarse grid in the lateral direction. We found that the prediction using the medium grid $(349 \times 23 \times 60)$ is just as good as that of the fine grid $(349 \times 45 \times 60)$, in contrast to the wind-tunnel data. So we finally used the medium grid $(349 \times 23 \times 60)$ as the default choice in the following simulations considering both solution accuracy and calculation time. To study grid independence, we 
also used the coarse grid to provide a comparison. The grid generation of the medium grid $(349 \times 23 \times 60)$ within and around cube arrays in the plane of $y / H=0$ and in the plane of $z / H=0$ is shown in Fig. 2a and b. In addition, we also studied a cube array of 14 rows and 21 rows using a similar grid structure to the medium $(349 \times 23 \times 60)$ and coarse $(235 \times 23 \times 47)$ grids for the seven-row cube array, generating grids for fourteen-row $(601 \times 23 \times 60$ and $415 \times 23 \times 47)$ and twenty-one-row $(853 \times 23 \times 60$ and $583 \times 23 \times 47)$ cube arrays.

For macroscopic simulations, we used the same computational flow domains as those in microscopic simulations but with a different grid structure. Considering the symmetrical characteristics, the three-dimensional (3D) microscopic urban canopy flows with groups of buildings are replaced by 2D macroscopic turbulent flows through continuous porous media. The distances above the porous region, from the domain inlet to the porous region, and from the porous region to the domain outlet, are the same as those in the microscopic simulations. For porous media corresponding to the cube cluster of seven rows, we used a coarse grid and a fine grid to investigate the grid independence problems, i.e. $191(x) \times 5(y) \times 71(z)$ and $266(x) \times 5(y) \times 86(z)$. For these two kinds of grids, a small grid size was used near the interfaces between the porous region and the surrounding clear fluid region for which the minimum grid sizes are 0.0035 and $0.0022 \mathrm{~m}$, respectively. As we found that numerical results using the two grids generated little difference, so the coarse grid is sufficient for the macroscopic simulation. For porous media corresponding to cube clusters of 14 and 21 rows, we used grids of $261(x) \times 5(y) \times 71(z)$ and $331(x) \times 5(y) \times 71(z)$. The grid number in macroscopic simulations can be reduced effectively because buildings disappear in porous media, and the grid size in a horizontal plane can be large in regions with small spatial gradients of variables, even larger than the size of buildings.

For both microscopic and macroscopic simulations using the porous turbulence model, we used a no-slip wall boundary condition at all wall surfaces and a normal zero-gradient boundary condition at the domain outlet, the domain top and all the symmetrical boundaries. At the upstream domain inlet, we used the power-law vertical profile of the mean streamwise velocity measured in the upstream free flow $\left(\bar{u}_{\text {inlet }}\right)$ and the calculated TKE $\left(\bar{k}_{\text {inlet }}\right)$ and its dissipation rate $\left(\bar{\varepsilon}_{\text {inlet }}\right)$ assuming an equilibrium turbulent flow as boundary conditions:

$$
\begin{aligned}
\bar{u}_{\text {inlet }}(z) & =U_{H}(z / H)^{0.16}, \\
k_{\text {inlet }}(z) & =u_{*}^{2} / \sqrt{C_{\mu}}, \\
\varepsilon_{\text {inlet }}(z) & =C_{\mu}^{3 / 4} k_{\text {inlet }}(z)^{3 / 2} /\left(\kappa_{v} z\right),
\end{aligned}
$$

where $C_{\mu}$ is $0.09, U_{H}=3.0 \mathrm{~m} \mathrm{~s}^{-1}, u_{*}=0.24 \mathrm{~m} \mathrm{~s}^{-1}, \kappa_{v}$ is von Karman's constant.

In the 'Ventair' code, the flow equations are expressed in time-implicit and conservative finite difference form on a staggered grid (see Eqs. 8 to 12a-12d). The transport equations for the momentum and turbulent properties were discretized by finite volume techniques. The hybrid upwind/central differencing scheme was used to discretize the advection terms, with an option of the second-order upwind scheme and the QUICK scheme. The discretized differential equations were solved by the SIMPLE algorithm. We used an under-relaxation factor of 0.5 for the mean velocities and pressure, and values of less than 0.5 for the turbulent properties to avoid divergence problems.

\subsection{Macroscopic Averaged Technique and Parameters in the Porous Model}

We regarded the urban canopy as spatially continuous porous media that acts as a sink/source of the momentum and turbulence properties of the urban flow. We used Eq. $7 \mathrm{~b}$ to calculate macroscopic time-averaged quantities by volume averaging the corresponding microscopic 
time-averaged quantities over an $\operatorname{REV}(\Delta V)$. The urban canopy consists of many units, each of which is formed by a building and a canyon. The definition of REV units should not be smaller than such a unit. We show two methods for the definition of the smallest REV units in such a seven-row cube array $(W=H)$; see Fig. 1c (Method 1) and 1d (Method 2). The Forchheimer coefficient $\left(C_{F}\right)$ shows the effect of form drag produced by building units on urban airflows, and may vary with the variation of vertical location $(z)$ and in different REV units. To analyse the Forchheimer coefficient $\left(C_{F}\right)$ using the high-resolution CFD simulation results for urban canopy flows, we defined a number of thin horizontal slabs centred at the height $z$ in this seven-row cube array, as shown in Fig. 1c; $d z$ is the interval distance between two neighbour grids in the vertical direction.

We defined the volume of a thin horizontal slab as $\Delta V=H\left(x_{i+1}-x_{i}\right) d z$, where $i$ is the number of the seven REV units (from 1 to 7). For Method 1, $x_{i}=2(i-1) H, x_{i+1}=2 i H$, and for Method 2, $x_{i}=2(i-1) H-0.5 H, x_{i+1}=2 i H-0.5 H$.

Important parameters for porous media include the porosity $(\phi)$, permeability $(K)$ and Forchheimer coefficient $\left(C_{F}\right)$. The porosity $(\phi)$ is defined in Eq. $2 \mathrm{a}$ and $2 \mathrm{~b}$ as the fraction of fluid volume in the total volume of an REV unit. The permeability $(K)$ in the Darcy terms for the viscous drag and the Forchheimer coefficient $\left(C_{F}\right)$ for the form drag can be approximately modelled by the Ergun equation as follows:

$$
\begin{aligned}
K & =\phi^{3} d_{p}^{2} /\left(150\left(1-\phi^{2}\right)\right), \\
C_{F} & =1.75 \beta / \sqrt{150 \phi^{3}},
\end{aligned}
$$

where $d_{p}$ is the characteristic size of solid particles in porous media, the porosity $\phi$ is 0.75 , $\beta=1$ in the original Ergun equation. Kanda (2006) studied the form drag in cube arrays of different building area densities (i.e. $1-\phi$ ) of a staggered order or aligned order using LES, and reported that the form drag in an aligned array is generally less than that in a staggered array. So we also revised the original Ergun equation, assuming $\beta=1.0$ (i.e. $C_{F}=0.22$ ) for the first row of building arrays and $\beta=0.56$ (i.e. $C_{F}=0.12$ ) for the other rows of such medium cube arrays according to Kanda (2006), considering the first row may provide shelter for the other rows and generate a larger form drag to the approaching flow than the others.

To obtain a better prediction of the form drag, the sectional Forchheimer coefficient $C_{F}(z)$ in each REV unit can be calculated from the pressure difference between the windward wall surfaces and the leeward wall surfaces of each building using,

$$
\begin{aligned}
C_{F}(z) & =\frac{f_{x}(z) \sqrt{K}}{\rho Q^{f}(z) u^{f}(z)}, \\
f_{x}(z) & =\frac{\int_{S} p(z) n_{x} d S}{\Delta V(z)},
\end{aligned}
$$

where $Q^{f}(z)$ and $u^{f}(z)$ are local intrinsic averages of time-averaged velocity and the streamwise velocity component in each thin horizontal slab of REV units $\left(Q^{f}=\sqrt{u^{f 2}+v^{f^{2}}+w^{f 2}}\right), K$ is the permeability, $f_{x}(z)$ is the sectional form (pressure) drag in each thin horizontal slab $(d S=d y d z, S=0.5 H d z$, the height of thin slabs is the local vertical grid size $d z$ ), and $\Delta V(z)=0.5 H^{2} d z$ is the total volume of each thin horizontal slab.

Therefore, the microscopic simulations were validated using wind-tunnel data. They were then were used to analyse the microscopic flow field within and around buildings, and to calculate the macroscopic time-averaged quantities and the sectional Forchheimer coefficient 

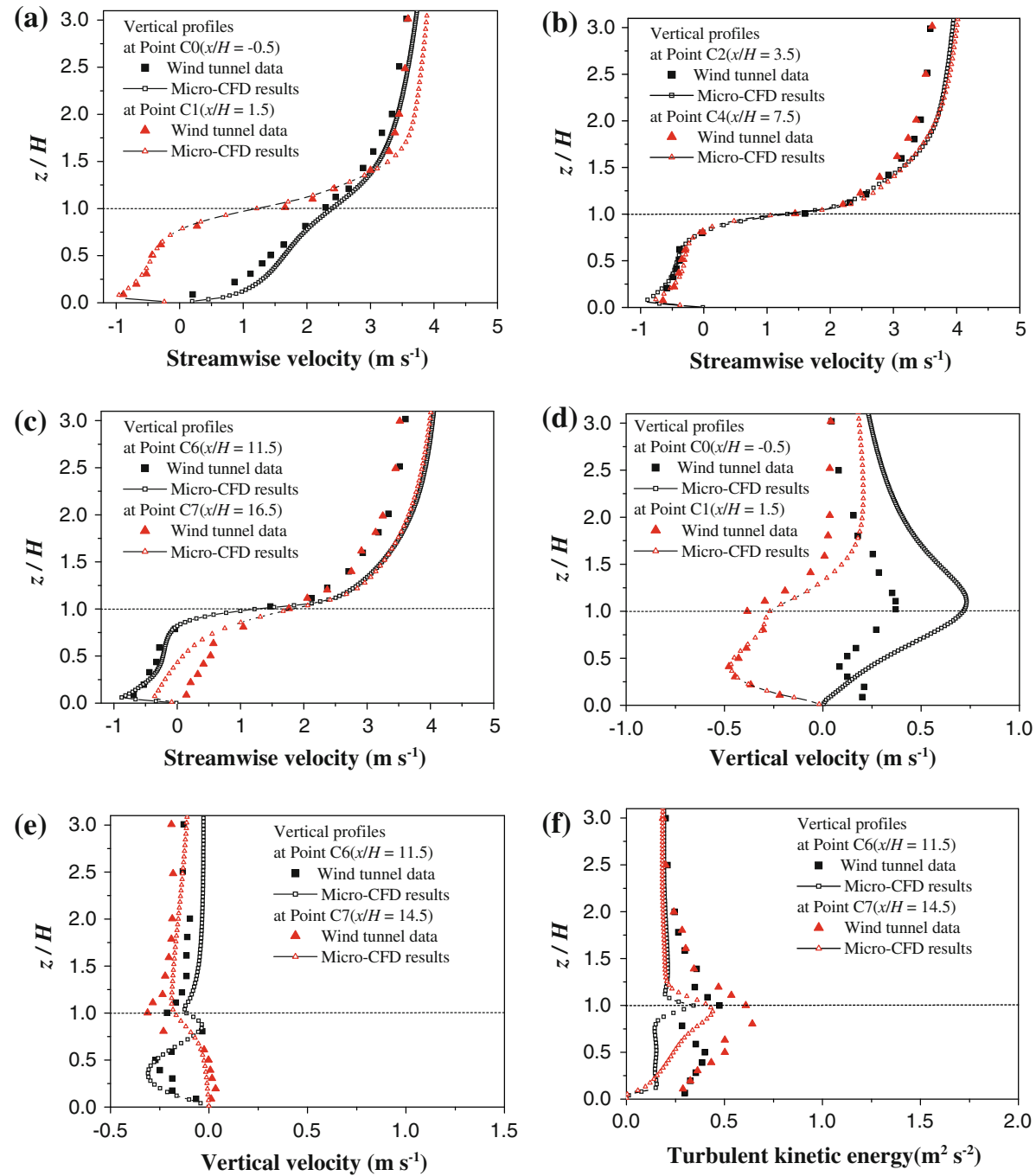

Fig. 3 Vertical profiles of streamwise velocity, vertical velocity and TKE at several points including both wind-tunnel data and microscopic simulation results in the seven-row cube array

in each thin slab of separated REV units. The macroscopic time-averaged quantities by integration of the microscopic simulation results show the macroscopic variation of the flow field and can be used for comparison with macroscopic simulation results using the porous turbulence model.

\section{Microscopic Simulation Results}

\subsection{Validation by Wind-Tunnel Data}

To validate the effectiveness of the present microscopic simulations $(349 \times 23 \times 60)$, Fig. 3 shows vertical profiles of the measured mean streamwise velocity, vertical velocity and TKE 
at several sample points. The present predictions are of similar accuracy as those in Lien and Yee (2004) and Santiago et al. (2007). Taken as a whole, we mainly emphasize the airflow below or near the roof level $(0<z / H<1.2)$ in cube arrays (i.e. Points $\mathrm{C} 1-\mathrm{C} 6)$. The mean streamwise velocity and vertical velocity were predicted generally well but TKE was underpredicted due to the limitation of the $k-\varepsilon$ turbulence model. The shapes of TKE profiles were well estimated, in contrast to wind-tunnel data. Using similar grids, we then studied the microscopic flow field in cube arrays of 14 rows and 21 rows with grids of $601 \times 23 \times 60$ and $853 \times 23 \times 60$, respectively.

Santiago et al. (2007) gave a detailed analysis of the flow mechanisms in such a 3D cube array. Here, we focused on the main flow pattern, ventilation conditions [i.e. flow rates and the air exchange rate $(\mathrm{ACH})]$ and the macroscopic flow reduction (spatially-averaged properties and drag effects of buildings) in such cube building arrays, which are useful for discussing the results of simulations using the porous turbulence model.

\subsection{Microscopic Flow Pattern}

The main streets and the secondary streets are parallel and perpendicular to the approaching flow, respectively. We define the secondary streets as 'canyons'. Figure 4 shows the distributions of the streamwise velocity, vertical velocity and TKE and velocity vectors in the plane $y / H=0$. Figure $4 d$ confirms that recirculation flows exist in each canyon, and that the vortices in canyons are not symmetric. Because of the clockwise characteristics of vortices in canyons, the mean vertical velocity is negative (i.e. downward motion) near the windward wall and positive (i.e. upward motion) near the leeward wall (see Fig. 4b). Such vertical mean flows contribute to the air exchange between street canyons below the roof level and the external flows above them. Due to the shelter effect of buildings, the mean streamwise velocity in canyons is much smaller than that above the roof level, resulting in a large velocity gradient and a strong shear layer near the roof level (see Fig. 4a). Such a strong shear layer contributes to large TKE near the roof level (see Fig. 4c).

Figure $5 \mathrm{a}$ and $\mathrm{b}$ shows the distribution of the streamwise velocity and TKE in the plane $z / H=0.8$; there is a reduction in the streamwise velocity and TKE from upstream regions to downstream regions of the cube array. Figure $5 \mathrm{c}$ and $\mathrm{d}$ shows velocity vectors in the plane $z / H=0.8$ and $z / H=0.2$; we find that recirculation flow exists in each canyon. At height $z / H=0.8$, the forward flow is deflected onto the windward corner of each building and some air enters the canyons laterally from the main street. However, in Fig. 5d, at height $z / H=0.2$, some air flows out of the canyons into the channels. Figure 5e shows the distribution of the spanwise velocity in the plane $y / H=-0.5$ (the interface plane between the main street and canyons); it confirms that the spanwise velocity is mainly positive (i.e. flow from the main street to canyons) in the upper half of the interface plane, and negative (i.e. from canyons to channels) in the lower half of the interface plane. Such flows contribute to lateral air exchanges between the main streets and canyons.

\subsection{Microscopic Flow Reduction and Ventilation Performance}

As shown in Fig. 5c, for the seven-row cube array, we defined six canyon units for the secondary streets and six channel units along the main street. Similarly, for fourteen-row and twenty-one-row cube arrays, 13 units and 20 units can be defined. In the canyons, 3D vortices exist and flow is relatively weak. Vertical (upward and downward) mean flows across canyon roofs and lateral mean flows across the interface between channels and canyons contribute to the ventilation and air exchange in canyons. In channels, the ventilation may be better 

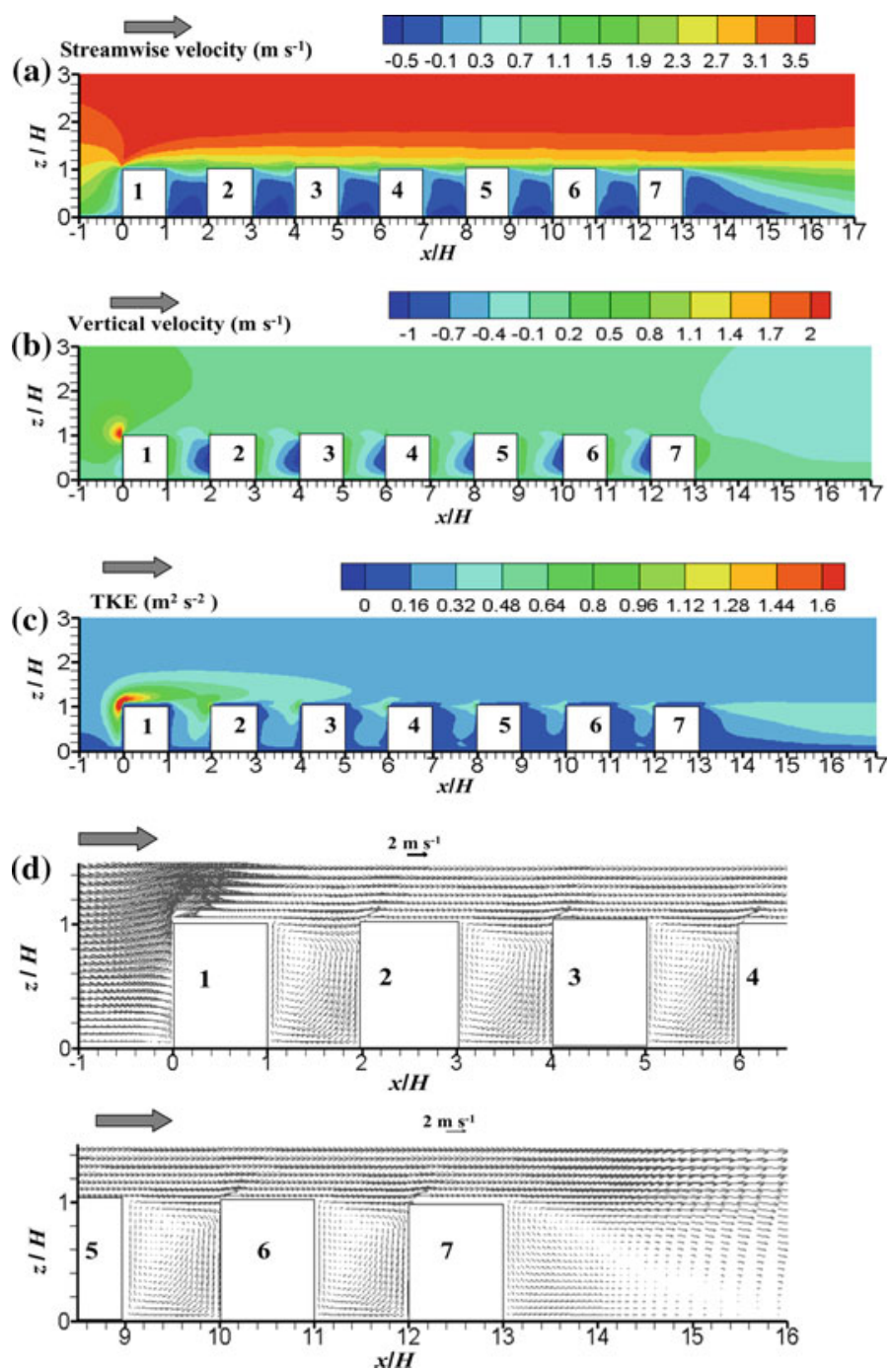

Fig. 4 Distribution of microscopic quantities in the seven-row cube array in the plane $y / H=0$ a streamwise velocity, b vertical velocity and c TKE. d Velocity vectors in the plane $y / H=0$

than that in canyons because the flow can pass easily through such aligned channels. Horizontal flow rates along the main street significantly determine the capacity of ventilation and pollutant dilution in channels. In addition, turbulence across all boundaries between a control volume (channels or canyons) and the external air environment also contributes to the ventilation of this control volume.

To quantify the flow reduction in the main street parallel to the approaching flow (i.e. through the channel units), we normalized the streamwise $(x)$ or vertical $(z)$ velocity components by the velocity $\left(U_{0}(z)\right)$ at the same height in the domain inlet. To estimate the ventilation flow rates, we defined the flow rate through the same area with the windward street entry far upstream (i.e. at the domain inlet here) as the reference flow rate $\left(Q_{\infty}\right)$ : 

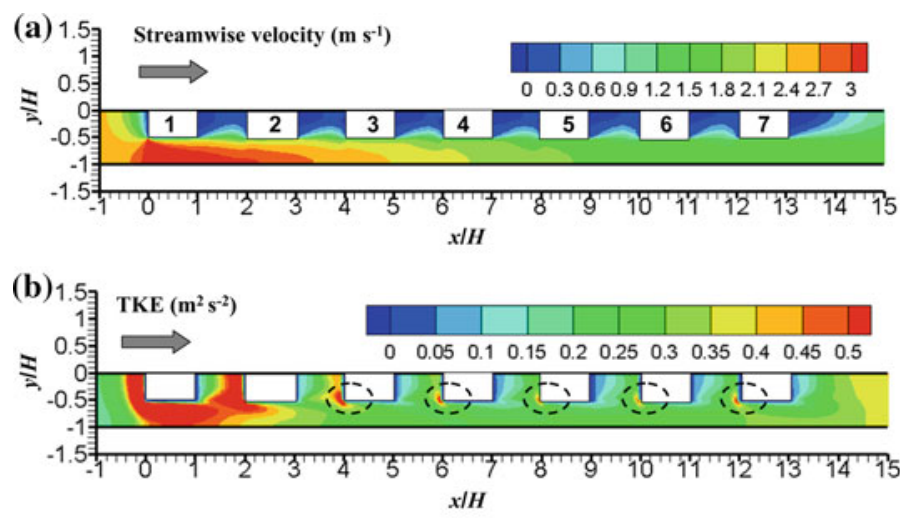

(c)

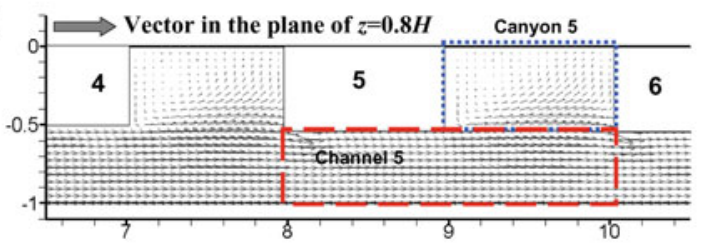

(d)
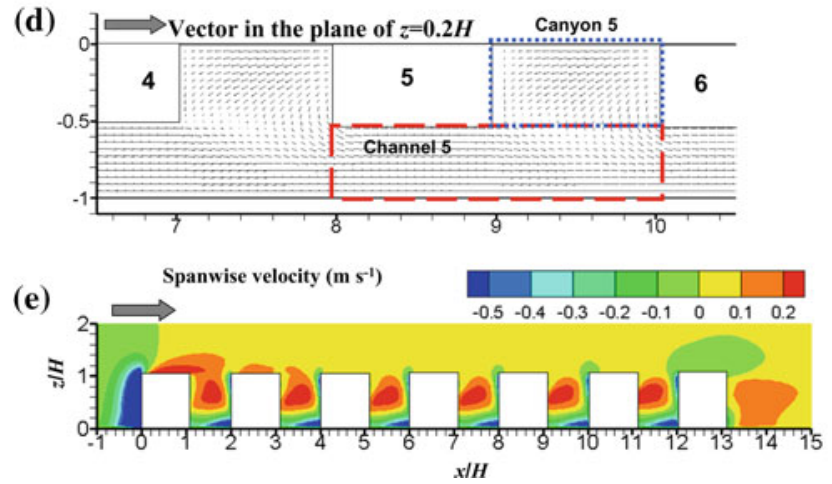

Fig. 5 Distribution of a streamwise velocity, $\mathbf{b}$ TKE in the plane $z / H=0.8$. Velocity vectors $\mathbf{c}$ in the plane $z / H=0.8$ and $\mathbf{d}$ in the plane $z / H=0.2$. e Distribution of spanwise velocity in the plane $y / H=-0.5$

$$
Q_{\infty}=\int U_{0}(z) d A,
$$

where $A$ is the area of the windward street entry of the building array, and $U_{0}$ is the streamwise velocity far upstream at the domain inlet.

Then we used this reference flow rate to normalize the volumetric flow rates along the main street (channels) or vertical flow rates across canyon roofs, as well as the effective flow rate through canyon roofs due to turbulence fluctuations, as defined in Hang et al. (2009).

$$
\begin{aligned}
Q^{*} & =\int_{A} \vec{V} \bullet \vec{n} d A / Q_{\infty}, \\
Q_{\text {turb } \pm}^{*} & = \pm \int 0.5 \sigma_{w} d A / Q_{\infty},
\end{aligned}
$$


where, in Eq. 18, $\vec{V}$ is the velocity vector, $\vec{n}$ is the normal direction of a given surface (street openings, a lateral face within channels, or canyon roofs), $A$ is the area of this given surface; and in Eq. 19, $A$ is the area of a given canyon roof $\left(A=H^{2} / 2\right.$ in this case), $\sigma_{w}=\sqrt{\overline{\overline{w^{\prime} w^{\prime}}}}=$ $\sqrt{2 k / 3}$ is the standard deviation of vertical velocity fluctuations on canyon roofs based on the assumption of isotropic turbulence $\left(u^{\prime}=v^{\prime}=w^{\prime}\right)$, and the given $u^{\prime}, v^{\prime}, w^{\prime}$ are the streamwise, spanwise, and vertical velocity fluctuations with $k=0.5\left(\overline{u^{\prime} u^{\prime}}+\overline{v^{\prime} v^{\prime}}+\overline{w^{\prime} w^{\prime}}\right)$ being the TKE. In Eq. 19, 0.5 $\sigma_{w}$ implies that turbulent exchange contributes to the same magnitude of upward and downward fluctuations across canyon roofs.

To evaluate the air exchange between each canyon unit and each channel unit, we introduced a concept of the air exchange rate $(\mathrm{ACH}$, i.e. the air change rate per hour). We first calculated the total volumetric flow rates due to mean flows and the total effective flow rates due to turbulent exchanges for each canyon unit and each channel unit, viz.

$$
\begin{aligned}
Q_{T \text { in }} & =\sum_{A_{i}} Q_{\text {in }}=-Q_{T \text { out }}=-\sum_{A_{i}} Q_{\text {out }}, \\
Q_{T \text { turb }} & =\sum_{A_{i}} Q_{\text {turb } \pm},
\end{aligned}
$$

where $A_{i}$ are surfaces of a given canyon or channel unit, $\sum_{A_{i}} Q_{\text {in }}$ (i.e. $Q_{T \text { in }}$, positive value) and $\sum_{A_{i}} Q_{\text {out }}$ (i.e. $Q_{T \text { out }}$, negative value) are the sums of inflow and outflow rates across these surfaces, and $\sum_{A_{i}} Q_{\text {turb }}$ is the sum of the effective flow rates due to turbulence across these surfaces.

To analyse the $\mathrm{ACH}$ for each of the canyon units and channel units, we defined a reference air exchange rate $\left(\mathrm{ACH}_{0}\right)$ in Eq. 22, and then utilized it to normalize all ACHs for each canyon unit and each channel unit in Eqs. 23 and 24. viz.

$$
\begin{aligned}
\mathrm{ACH}_{0} & =3600 Q_{\infty} /\left(\operatorname{vol}_{\text {canyon }}+\operatorname{vol}_{\text {channel }}\right), \\
\mathrm{ACH}^{*} & =3600 Q_{T \text { in }} /\left(\operatorname{vol} \mathrm{ACH}_{0}\right), \\
\mathrm{ACH}_{\text {turb }}^{*} & =3600 Q_{T \text { turb }} /\left(\operatorname{vol} \mathrm{ACH}_{0}\right),
\end{aligned}
$$

where vol canyon and vol $_{\text {channel }}$ are the volumes of each canyon unit or channel unit, and $\mathrm{ACH}^{*}$ and $\mathrm{ACH}_{\text {turb }}^{*}$ are the normalized $\mathrm{ACH}$ s for each given canyon unit or channel unit due to all the volumetric flow rates $\left(Q_{T \text { in }}\right)$ and due to the total effective flow rates by turbulent exchanges $\left(Q_{T \text { turb }}\right)$ across the surfaces of a local unit. Finally, vol is the control volume of each canyon unit or each channel unit.

Figure $6 \mathrm{a}$ and $\mathrm{b}$ shows horizontal profiles of the normalized streamwise velocity and the normalized vertical velocity along the street centrelines $(z=H$ or $0.5 H)$ of the main street for three cube arrays of 7, 14 and 21 rows. Figure 6c displays the normalized horizontal flow rate along the main street in cube arrays of 7,14 and 21 rows; $x / H=0$ is the windward street entry. We find that the normalized streamwise velocity and the normalized horizontal flow rate at $x / H=0$ is a little more than 1.0, denoting that flow at the windward entry is a little stronger than that at the domain inlet far upstream. After an accelerating process across the windward street entry, the normalized streamwise velocity and the normalized horizontal flow rate decreases along the main street because some air parcels leave the main street upward, as can be confirmed by the positive vertical velocity in Fig. 6b. At places with a sufficient distance far from the two entries of the main street (windward and leeward entries), the macroscopic variation of the streamwise velocity and horizontal flow rate is small (see Figure 6a, c). In such regions, it is interesting to find that the horizontal profile of the vertical velocity (see Fig. 6b) at the roof level $(z=H)$ is a relatively smooth shape and nearly remains 
(a)
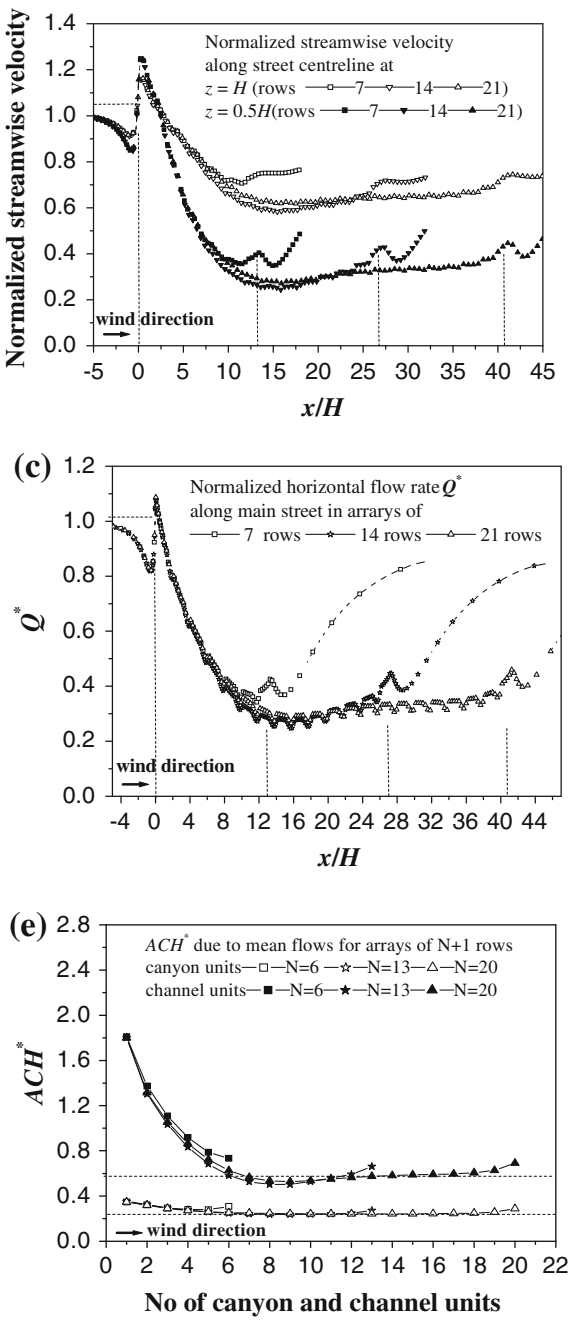

(b)
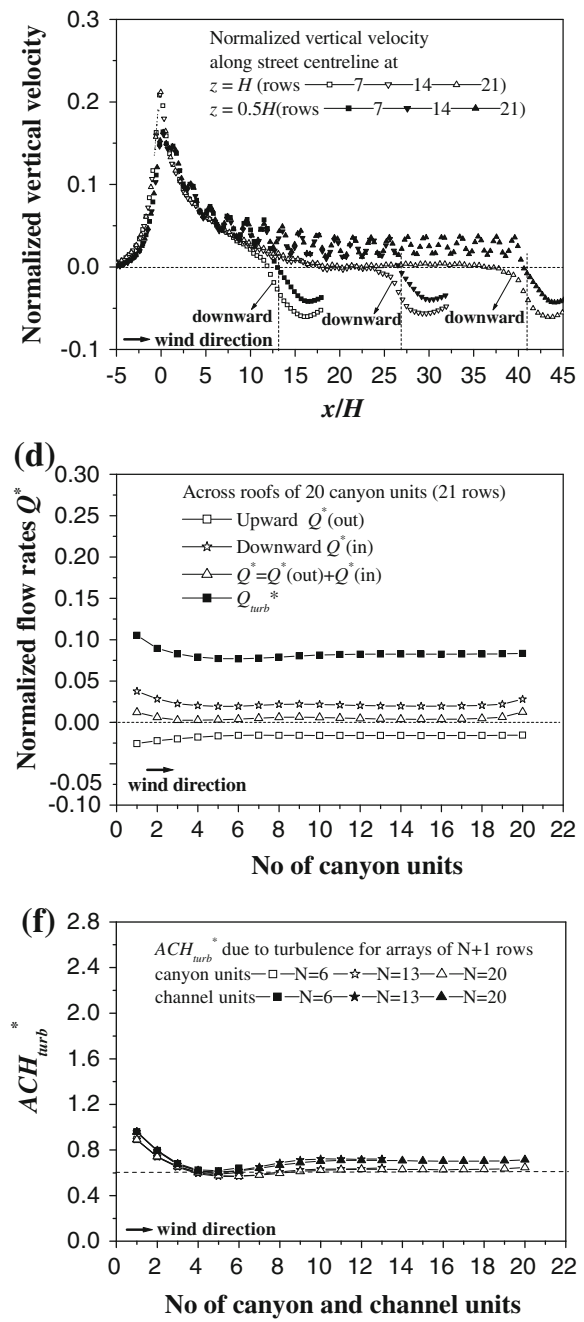

Fig. 6 Horizontal profiles of a normalized streamwise velocity and b normalized vertical velocity along street centrelines of the main street parallel to upstream wind for three cube arrays of 7, 14 and 21 rows, c normalized horizontal flow rate along the main street for three cube arrays of 7, 14 and 21 rows. $\mathbf{d}$ Normalized flow rates across roofs of 20 canyon units in the 21 row cube array. Normalized ACHs of canyon units and channel units in cube arrays of 7,14 and 21 rows e due to the flow rates (mean flow) and $\mathbf{f}$ due to the effective flow rates (turbulent exchange). In $\mathbf{a}-\mathbf{c}, x / H=0$ is the windward street entry

at zero; however, that at $z=40.5 H$ shows a variation of a periodic wave shape with positive values (i.e. upward motion). The profile of horizontal flow rates along the main street also shows a similar wave shape (see Fig. 6c), and may result from the deflection of the forward flow on the windward corner of buildings and the lateral air exchange between the channel units and canyon units. Near the leeward street entries (i.e. $x / H=13,27$ and 41 for cube arrays of 7, 14 and 21 rows), the normalized vertical velocity is negative (see Fig. 6b), which confirms a downward flow rate from the above external flow into the main street across the 
street roof near the leeward entry. As a result, the streamwise velocity and the horizontal flow rate increase a little along the main street near the leeward entry (see Fig. 6a, c). In a macroscopic view, as the cube array changes from 7 rows to 14 rows, the horizontal flow rate continues to decrease along the main street. However, as the cube array changes from 14 rows to 21 rows, the horizontal flow rate ceases to decrease.

Figure $6 \mathrm{~d}$ shows the normalized flow rates across roofs of the 20 canyon units in the twentyone-row cube array due to upward and downward flows as well as turbulent exchanges. The flow rate due to the downward inflow $Q^{*}$ (in) (i.e. positive) is larger than that due to the upward outflow $Q^{*}$ (out) (i.e. negative), so the total vertical flow rate $\left(Q^{*}=Q^{*}\right.$ (out) $+Q^{*}($ in $\left.)\right)$ is positive (i.e. downward inflow). This result shows that the flow rates entering canyons are a little more than those leaving them across their roofs, and they differ from $2 \mathrm{D}$ turbulent canyon flows in which $Q^{*}$ (out) is always equal to $Q^{*}$ (in). The extra vertical inflow rate across canyon roofs may supply external clean air from external flows downward into 3D canyons. The effective flow rate due to turbulent exchanges $\left(Q_{\text {turb }}^{*}\right)$ across canyon roofs exceeds several times that due to vertical mean flows, confirming that turbulence is very important for the ventilation of canyons. Figure 6e and $\mathrm{f}$ shows the normalized ACHs for each canyon unit and each channel unit in cube arrays of 7,14 and 21 rows (6,13 and 20 units) due to the mean flow and turbulence. We find that the normalized ACHs (see Fig. 6e) due to the mean flow in channel units are almost three times those in canyon units, because the horizontal flow rates through channels (the main street) are several times the flow rates for canyons. Air exchange rates due to turbulence (see Fig. 6f) in canyon units are of the same order as those in channel units. For canyon units, the normalized ACHs due to turbulence are about 0.6 (see Fig. 6f) and those due to the mean flow are about 0.2 (see Fig. 6e), showing that turbulence is even more important than the mean flow for air exchanges in canyon units. For air exchanges in the channel units (see Fig. 6e, f), turbulence also contributes significantly, in contrast to the mean flows.

\subsection{Spatially-Averaged Quantities in REV Units and Sectional Forchheimer Coefficient}

There are two methods of REV definition (Method 1 and Method 2) in the volume-averaging technique (see Fig. 1c, d). Using both methods, Fig. 7a-c shows the horizontal profiles of normalized spatially-averaged streamwise velocity, vertical velocity and TKE (i.e. macroscopic quantities) at $z=0.5 H$ (thin horizontal slabs), and the sectional Forchheimer coefficient at $z=H$ in cube arrays of 7,14 and 21 REV units. $U_{0}(z)$ is the streamwise velocity at the upstream domain inlet at the same height $(z=0.5 H)$. It is confirmed that these three macroscopic quantities and the Forchheimer coefficients calculated using two different methods of REV definition are almost the same in most locations, except at the first unit (No. 1) and the final unit (Nos. 7, 14, or 21). This is usual the airflow on the windward side of the first cube and in the wake region behind the final cube is different from that in canyons between two neighbouring cubes.

Figure 7e shows the vertical profiles of the sectional Forchheimer coefficient for the seven REV units in the seven-row cube array. We find that the Forchheimer coefficient at unit 1 (i.e. the first building) is greatest at most vertical locations because the first cube blocks the approaching flow the most effectively. The first cube provides a significant 'shelter' for the second cube (i.e. unit 2) and, as a result, the Forchheimer coefficient due to the blockage of the second cube is the smallest. For all seven units, the Forchheimer coefficient at roof level (i.e. $z=H$ ) is smaller than that at a lower level ( $z / H$ from 0.35 to 0.95 ). Both Fig. $7 \mathrm{~d}$ and e confirm that the Forchheimer coefficient for the seven units of the seven-row cube 

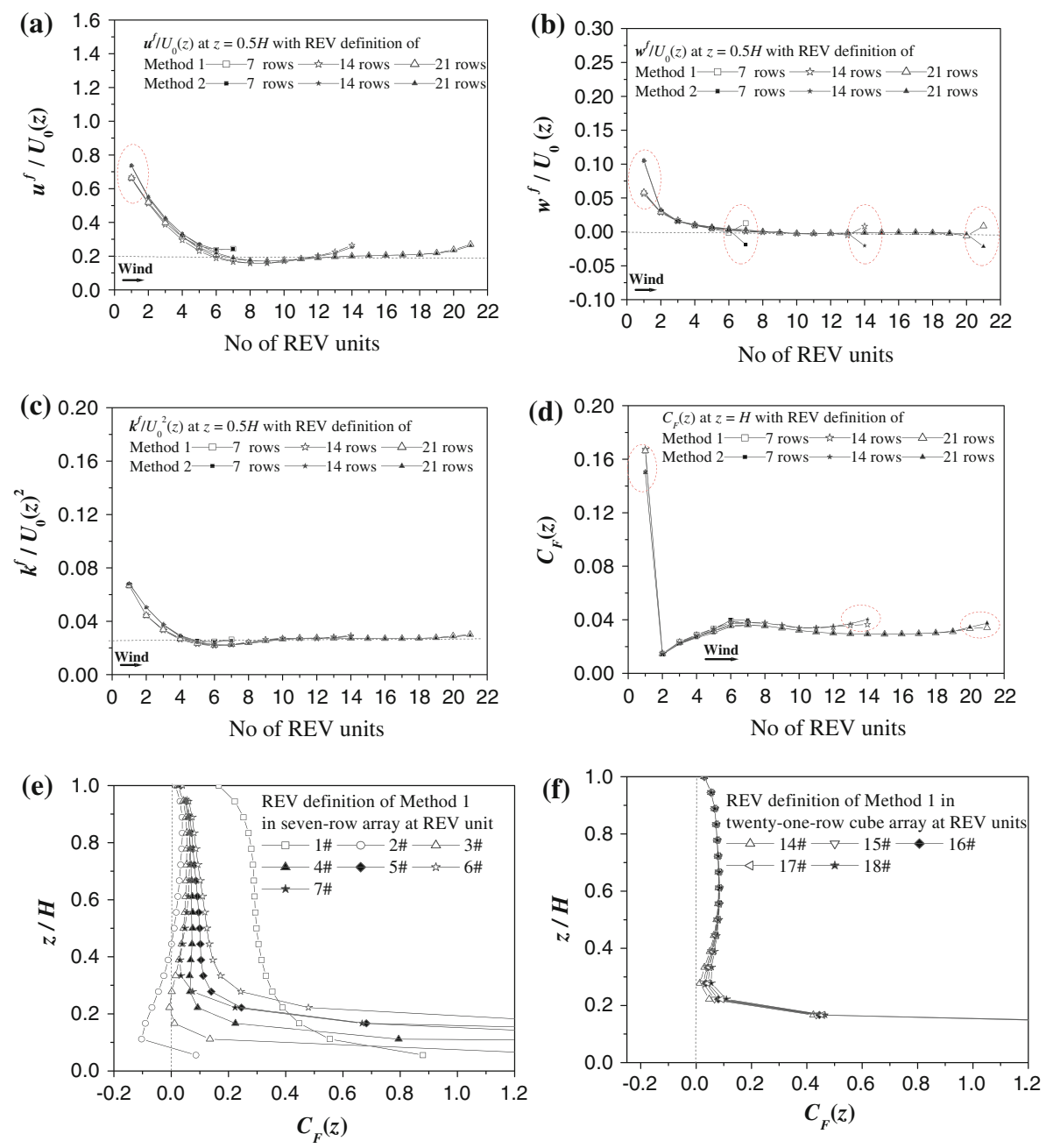

Fig. 7 Horizontal profiles of macroscopic quantities of a streamwise velocity, b vertical velocity, $\mathbf{c}$ TKE at $z=0.5 H$ (thin horizontal slabs), and $\mathbf{d}$ sectional Forchheimer coefficient $\left(C_{F}\right)$ at $z=H$ with 7,14 and $21 \mathrm{REV}$ units using two methods of REV definition. Vertical profiles of $C_{F}(z)$ : $\mathbf{e}$ for the seven units in the seven-row cube array and $\mathbf{f}$ for the five REV units Nos. 14-18 in the twenty-one-row cube array. In a-c, $U_{0}$ is streamwise velocity at the upstream domain inlet at the same height $(z=0.5 H)$. Method 1 and Method 2 are shown in Fig. 1c and 1d as two methods of REV definition. All results are from microscopic solution using grids of $349 \times 23 \times 60,602 \times 23 \times 60$ and $853 \times 23 \times 60$ for these three cube arrays

array always changes from No. 1-7. However, Fig. 7d shows that the horizontal profile of the Forchheimer coefficient at roof level from unit 12 to unit 18 almost remains constant. To verify whether the sectional Forchheimer coefficient from units 12 to 18 change little at other heights, Fig. $7 \mathrm{f}$ displays the vertical profiles of the Forchheimer coefficient at these units, finding that it varies very little.

Using the sectional Forchheimer coefficient distribution calculated in microscopic simulations, we performed macroscopic simulations using the porous turbulence model. Since the spatially-averaged variables using the two methods of REV definition are approximately 

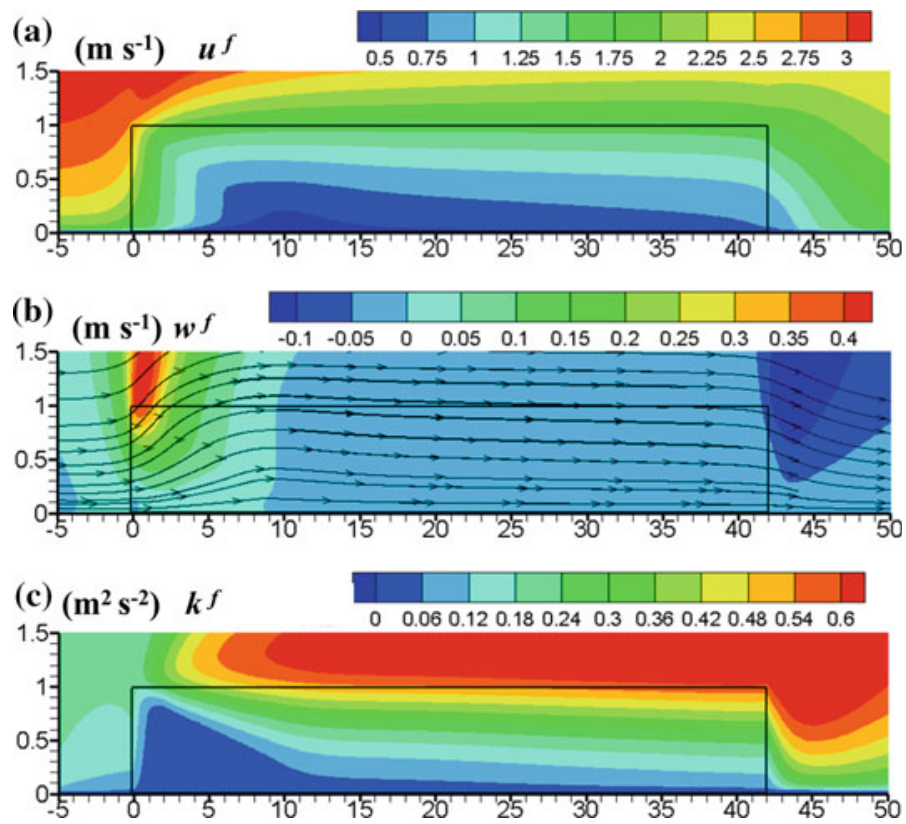

Fig. 8 Distribution of a macroscopic streamwise velocity, $\mathbf{b}$ macroscopic vertical velocity, and $\mathbf{c}$ macroscopic TKE for porous media of a twenty-one-row cube array, using the revised Ergun equation to calculate the Forchheimer coefficient $C_{F 1}$ (in Eq. $14 \mathrm{a}, 14 \mathrm{~b}$, for unit $1, \beta=1, C_{F}=0.22$; for all other REV units, $\beta=0.56$, $\left.C_{F}=0.12\right) . x=0$ is the windward edge of the porous region

the same except for those at the first and final units, we used only Method 1 in the following analysis and discussion.

\section{Macroscopic Simulation Results}

\subsection{Characteristics of Macroscopic Porous Flows}

Using the revised Ergun equation for the Forchheimer coefficient (see Eq. 14a, 14b, $\beta=0.56$ for all REV units except unit 1), Fig. 8 shows the distribution of macroscopic streamwise velocity, vertical velocity and TKE for the twenty-one-row porous media. Figure 9 shows horizontal profiles of macroscopic variables at different heights in porous media of the seven-row and twenty-one-row cube arrays, in which $U_{0}(z)$ is the streamwise velocity at the upstream domain inlet $(z / H=1.0,0.5,1 / 6$, respectively) and $x=0$ is the windward edge of the porous region.

On the one hand, the macroscopic flow pattern is similar to the microscopic flow pattern. The drag effect of porous media reduces the flow on the windward side of the porous region $(x / H<0)$ and the flow reduction region $(0<x / H<10)$. The macroscopic vertical velocity is positive (i.e. upward motion) and the macroscopic streamwise velocity decreases in these two regions (see Fig. 9a, b). The macroscopic vertical velocity is greatest near the windward edge of the porous region (see Fig. 9b) and then decreases from $x=0$ to $x=10 H$. In the vicinity of the leeward edge of the porous region (near and behind $x / H=42$ ), the resistance of porous media disappears, and turbulent shear stress transports more momentum 
Fig. 9 Comparisons of macroscopic simulation results for porous media of the seven-row and twenty-one-row cube array using $C_{F 1}$ (for unit 1 , $\beta=1, C_{F}=0.22$; for all other REV units, $\beta=0.56$,

$C_{F}=0.12$ ):

a streamwise velocity, b vertical velocity and c TKE. $U_{0}(z)$ is the streamwise velocity at the upstream domain inlet at the same height $(z / H=1.0,0.5,1 / 6$ respectively). $x=0$ is the windward edge of the porous region
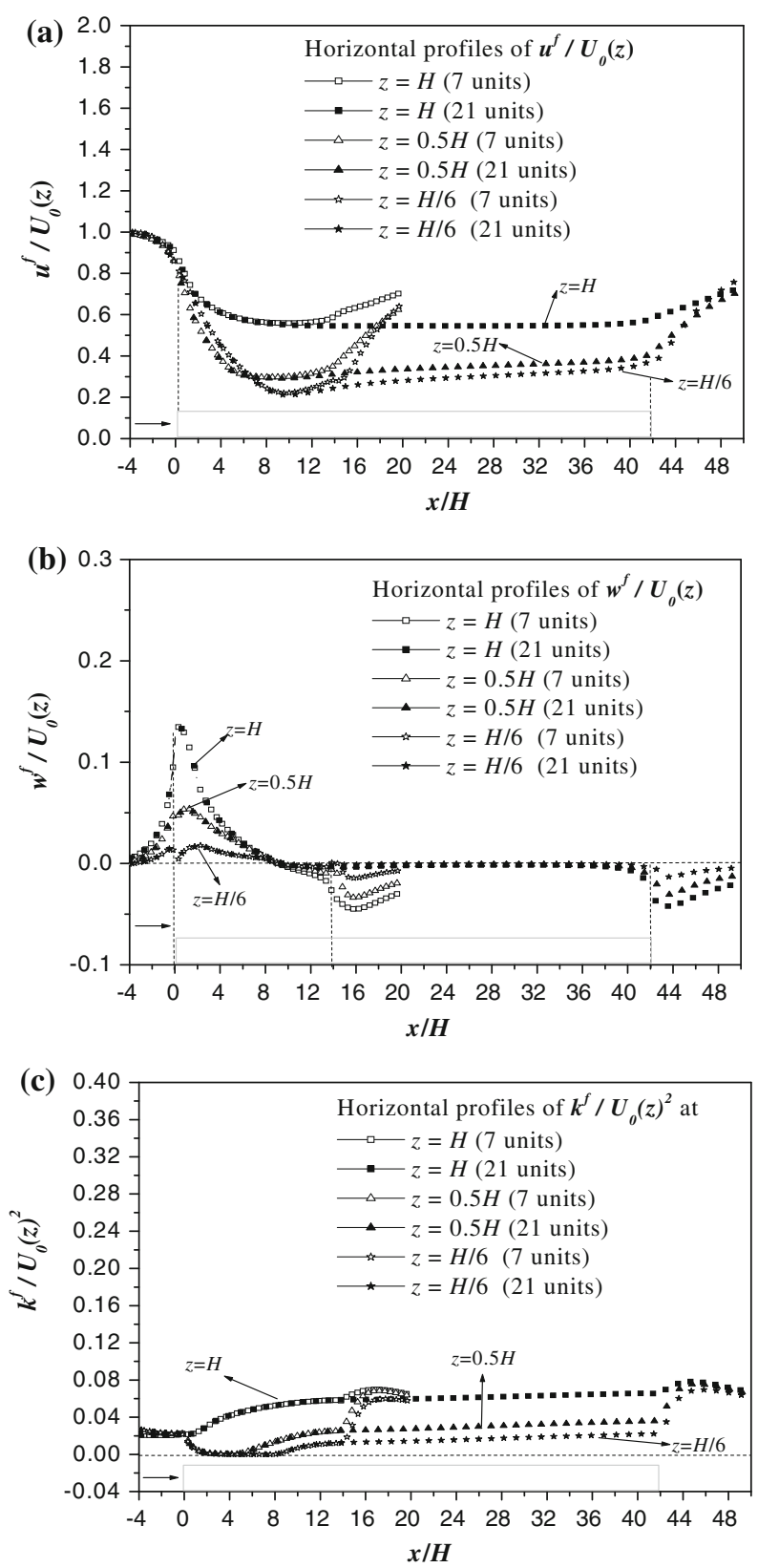

downward. The downward flow (i.e. negative vertical velocity) brings air into the porous region (see Fig. 9b) and, as a result, the macroscopic streamwise velocity increases a little (see Fig. 9a).

On the other hand, the macroscopic and microscopic flow regimes are different. In microscopic simulations, we confirmed that such 3D turbulent flows include the channel flow regime along the main street and the skimming flow regime in canyons. However, in macro- 
scopic simulations, the difference in the main street and canyons disappears because all the microscopic time-averaged variables are spatially averaged by the volume-averaging technique. More importantly, in the microscopic flow field, the windward edge of the first cube is an important source of TKE resulting in strong turbulence in that region (see Figs. 4c, $5 \mathrm{~b})$. However, the windward boundary of the porous region in macroscopic simulations is a strong sink (i.e. the Darcy term and the Forchheimer term) for turbulence, so TKE near the windward edge of the porous region is small. In the wake region of microscopic simulations, recirculation flows exist behind the final cube (see Fig. 4d). However, on the leeward side of the porous region in the macroscopic flow field (see Fig. 8) the recirculation disappears due to the volume-averaging technique in the porous region.

Above the top interface of the porous region and near the leeward edge of the porous region, TKE is large (see Fig. 8c). There is a shear layer near the interface between the porous region and the overlying clear fluid region. The downward transport of momentum due to turbulent shear stress acts as a motor for the flow through porous media, and the drag effects (i.e. the Darcy and Forchheimer terms) in the porous region contribute to the removal of momentum there. If the porous region is sufficiently long, a balance may be established in the macroscopic fully developed region. Figure 9 confirms that a macroscopic fully developed region is approximately generated at places far from the two ends in the longest porous media (21 REV units), where the macroscopic streamwise velocity almost remains constant, the macroscopic vertical velocity is nearly zero, and TKE increases at a low rate.

\subsection{Effect of Different Terms in the Porous Turbulence Model}

In Eqs. 8 to 12a-12d, we qualitatively analysed the effect of all Darcy and Forchheimer terms that appear in the porous turbulence model. Here, we quantitatively analyse these terms using macroscopic simulation results. First, for the transport of macroscopic streamwise velocity (see Fig. 10), both the Darcy (udarcy) and the Forchheimer terms (uForch) are negative, acting as sink terms for the momentum transport. The magnitudes of these two terms are large
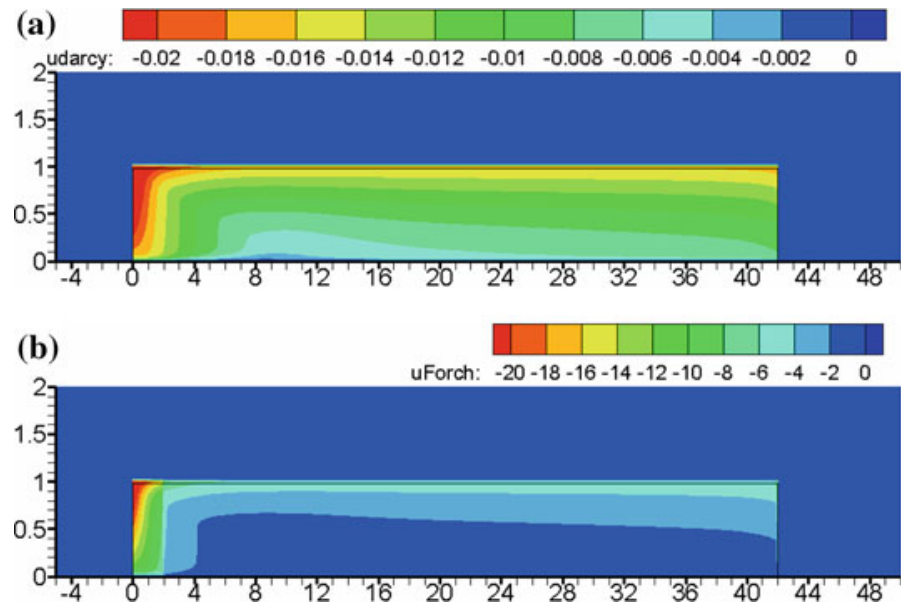

Fig. 10 Effects on the macroscopic streamwise velocity in porous media of the twenty-one-row cube array using $C_{F 1}$ (for unit $1, \beta=1, C_{F}=0.22$; for all other REV units, $\beta=0.56, C_{F}=0.12$ ) due to a the Darcy term (udarcy), and $\mathbf{b}$ the Forchheimer term (uForch). $x=0$ is the windward edge of the porous region. The two terms appear in Eq. 9. $x / H$ from 0 to 42 is the porous region of $21 \mathrm{REV}$ units 

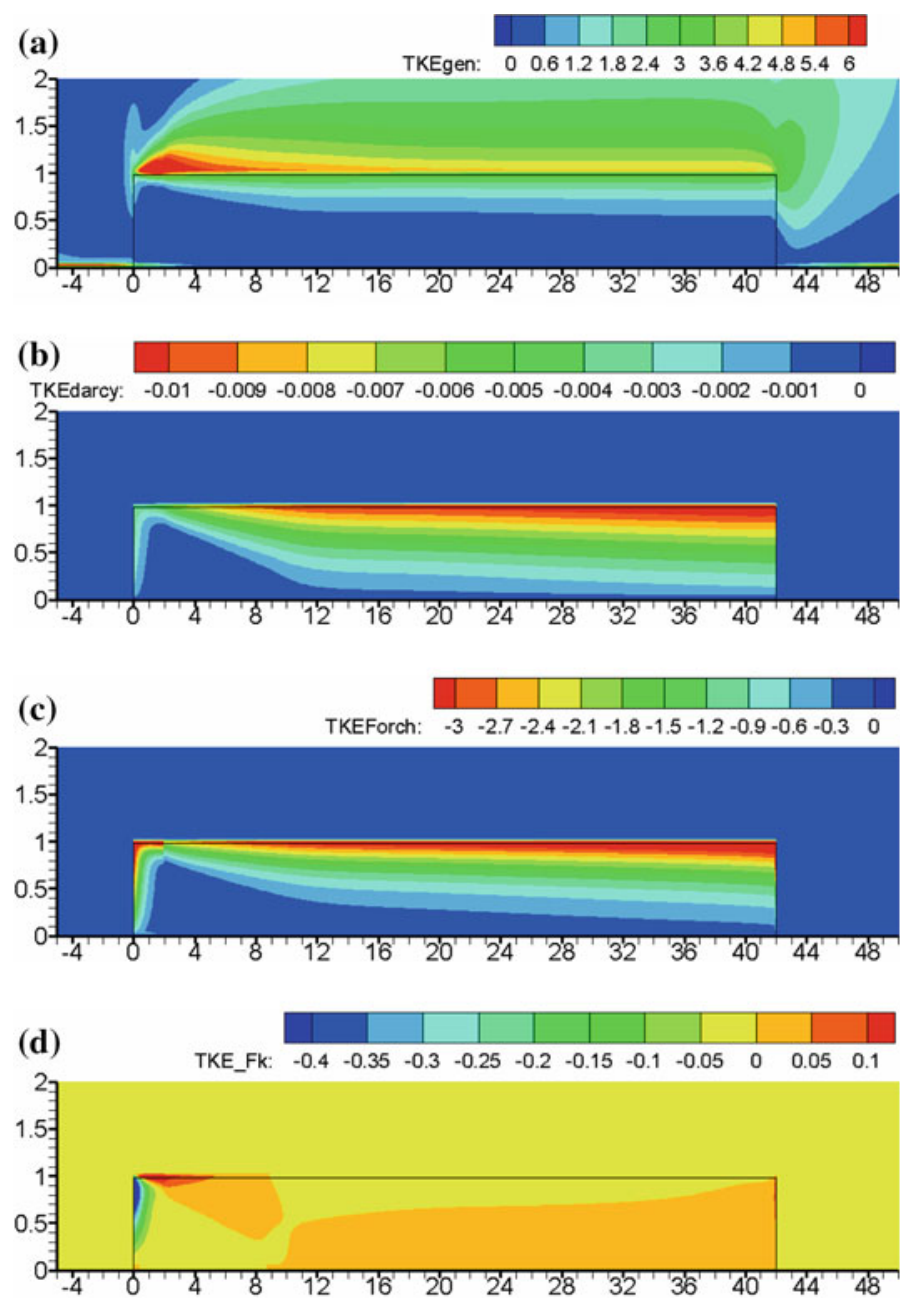

Fig. 11 Effects on the macroscopic TKE in porous media of the twenty-one-row cube array using $C_{F 1}$ (for unit $1, \beta=1, C_{F}=0.22$; for all other REV units, $\beta=0.56, C_{F}=0.12$ ) due to a the generation term of TKE (TKEgen), b the Darcy term (TKEdarcy), and the two Forchheimer terms of c TKEForch and d TKE_Fk. $x=0$ is the windward edge of the porous region. The three terms appear in Eq. $10 . x / H$ from 0 to 42 is the porous region of $21 \mathrm{REV}$ units

near the windward edge of the porous region where the macroscopic streamwise velocity is large. The Forchheimer term (i.e. of the order of 10) is much larger than the Darcy term (i.e. of the order of 0.01). In high-Reynolds-number flow in porous urban canopies, the form drag due to the resistance from buildings dominates the macroscopic velocity reduction, in contrast to the viscous drag (the Darcy term), because the fluid viscosity $(\mu)$ is small.

Figure 11 shows the TKEgen, TKEdarcy, TKEForch and TKE_Fk terms for the transport equation of macroscopic TKE. We find that the TKEgen term near the interface between the porous region and the overlying clear fluid region is the main source of turbulence generation, resulting in a shear layer near the interface. There is a large gradient of turbulence near the interface. We also find that the magnitude of the Forchheimer term of TKE (TKEForch, of the order of 1) exceeds that of the Darcy term (i.e. TKEdarcy, of the order of 0.01) by about 

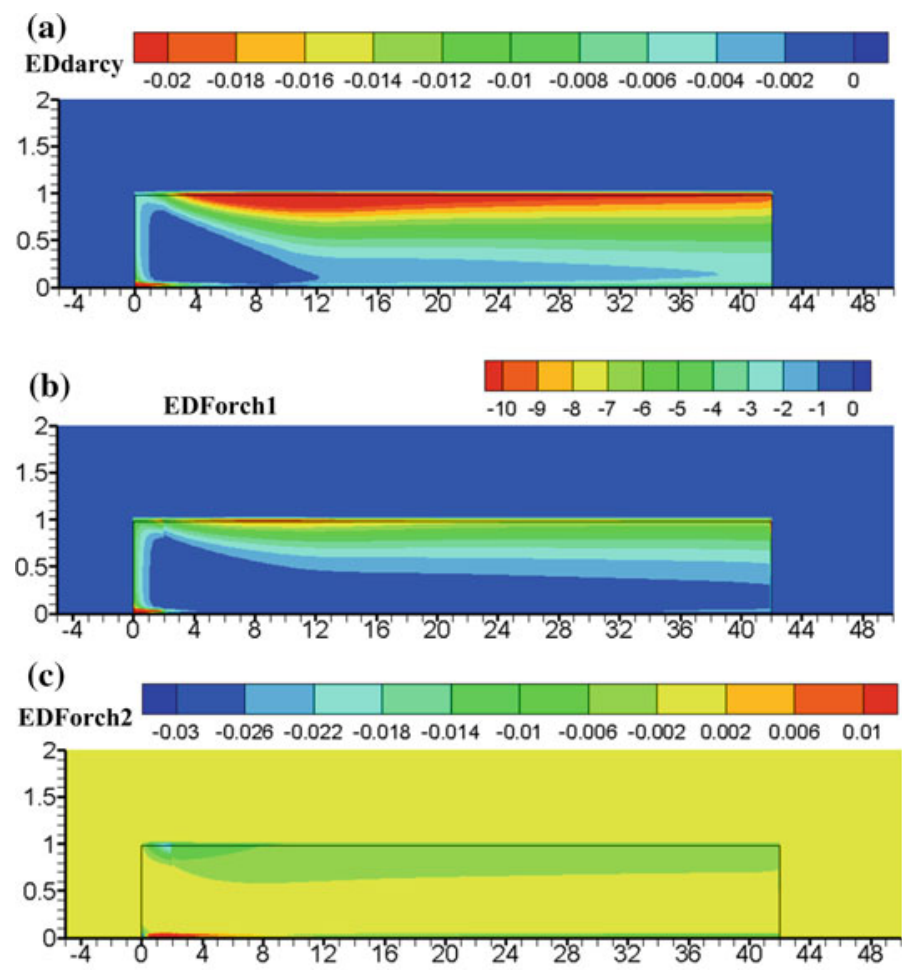

(d)

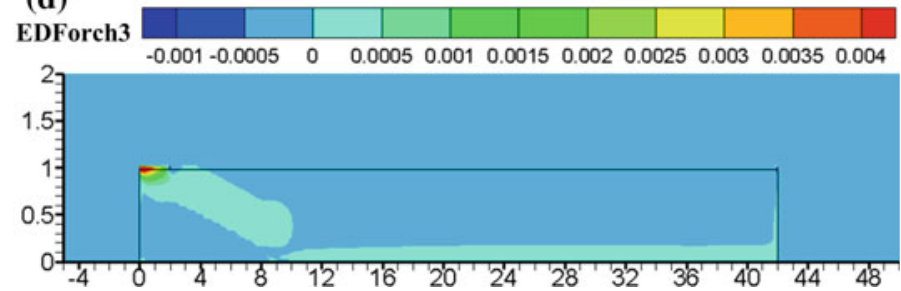

Fig. 12 Effects on the macroscopic dissipation rate of TKE in porous media of the twenty-one-row cube array using $C_{F 1}$ (for unit $1, \beta=1, C_{F}=0.22$; for all other REV units, $\beta=0.56, C_{F}=0.12$ ) due to a the Darcy term (EDdarcy), the three Forchheimer terms of $\mathbf{b}$ EDForch1, $\mathbf{c}$ EDForch2, and $\mathbf{d}$ EDForch3. $x=0$ is the windward edge of the porous region. All these terms are shown in Eq. 11. $x / H$ from 0 to 42 is the porous region of 21 REV units.

a hundred times. The TKEForch and TKEdarcy terms are negative, confirming a significant contribution to the reduction of turbulence, especially near the windward edge of the porous region. The TKE_Fk term (see Fig. 11d) is negative at the windward edge of the porous region $(x=0)$ and is positive near the upstream region of the top interface $(x / H$ from 0 to $4, z=H)$, showing that this term may act as either a source or sink term for turbulence generation.

Figure 12 shows the Darcy term (EDdarcy) and all Forchheimer terms (EDForch1, EDForch2, EDForch3) for the transport equation of the macroscopic dissipation rate of TKE (see Eq. 11). Similarly to that described in the previous paragraph, both the Darcy term (EDdarcy) and the EDForch1 term are negative, i.e. they weaken the dissipation rate of TKE. And the EDForch1 term (of the order of 1) significantly exceeds the EDdarcy term (of the 

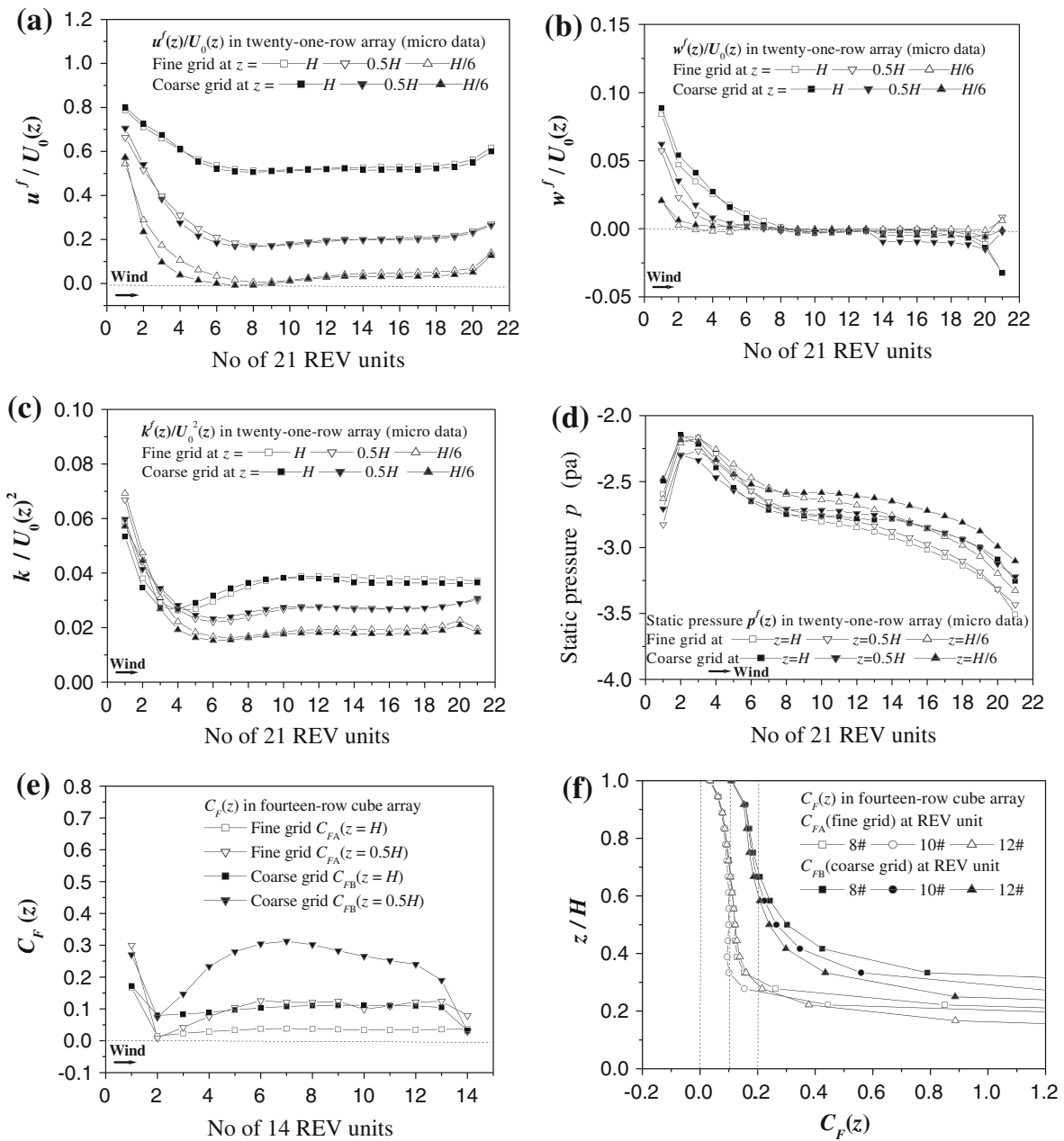

Fig. 13 Comparison of spatially-averaged variables of a streamwise velocity, b vertical velocity, $\mathbf{c}$ TKE, and d static pressure for the twenty-one-row cube array. Distributions of sectional Forchheimer coefficient for the fourteen-row cube array e horizontal profiles at different heights, and $\mathbf{f}$ vertical profiles at REV units Nos. 8, 10 and 12 . Fine and coarse grids were used in microscopic simulations. $U_{0}(z)$ is the streamwise velocity in the free flow far upstream at the height $z$

order of 0.01) in decreasing the reduction of TKE. In addition, the other two Forchheimer terms (EDForch2 and EDForch3) may increase or decrease the dissipation rate of TKE, but their magnitudes (of the order of 0.01 or 0.001 ) are negligible compared to that of EDForch1 because the fluid viscosity $\mu$ in EDForch2 and EDForch3 is small. So the difference in the EDForch2 and EDForch3 terms between Eq. 11 used here and the original equation derived by Antohe and Lage (1997) is negligible.

\subsection{Comparison of Macroscopic Quantities in Microscopic and Macroscopic Results}

To evaluate the grid independence of macroscopic quantities in microscopic simulation results, Fig. 13a-d shows horizontal profiles of spatially-averaged variables (i.e. macroscopic 
streamwise velocity, vertical velocity, TKE and static pressure) at different heights in the 21row cube array using the fine grid $(853 \times 23 \times 60)$ and the coarse grid $(583 \times 23 \times 47)$. We find that the macroscopic velocity components and turbulence are similar using the two grids, but the pressure using the two grids is a little different in the $21 \mathrm{REV}$ units. This confirms that static pressure is grid sensitive in these simulations. As a result, as shown in Fig. 13e and $\mathrm{f}$, the sectional Forchheimer $\left(C_{F}\right)$ coefficient is also grid sensitive. For $C_{F}(z)$ in the fourteen-row cube array from microscopic simulation results using the fine grid $(601 \times 23 \times 60)$ and the coarse grid $(415 \times 23 \times 47)$, we named this as $C_{F A}$ (fine grid) and $C_{F B}$ (coarse grid), respectively. The constant Forchheimer $\left(C_{F}\right)$ coefficients calculated by the revised Ergun equation ( $\beta=0.56, C_{F}=0.12$ for all the REV units except unit 1 in Eq. $14 \mathrm{a}, 14 \mathrm{~b}$ ) and the original Ergun equation ( $\beta=1.0, C_{F}=0.22$ in Eq. $\left.14 \mathrm{a}, 14 \mathrm{~b}\right)$ were defined as $C_{F 1}\left(\beta=0.56, C_{F}=0.12\right)$ and $C_{F 2}\left(\beta=1.0, C_{F}=0.22\right)$. Here, $C_{F B}$ and $C_{F 2}$ are larger than $C_{F A}$ and $C_{F 1}$.

Then we compare macroscopic quantities predicted by microscopic and macroscopic simulation results for the fourteen-row cube array. For microscopic predictions, two groups of spatially-averaged quantities were used, i.e. those calculated using the fine grid $(601 \times 23 \times 60)$ and those using the coarse grid $(415 \times 23 \times 47)$. For macroscopic simulations using the porous turbulence model, we performed the predictions with four kinds of Forchheimer coefficients, i.e. the sectional Forchheimer coefficients $\left(C_{F}(z)\right)$ from microscopic simulation results using the fine grid $\left(C_{F A}\right)$ and the coarse grid $\left(C_{F B}\right)$, constant Forchheimer coefficients (see Eq. $14 \mathrm{a}, 14 \mathrm{~b})$ using the revised or original Ergun equation, i.e. $C_{F 1}\left(\beta=0.56, C_{F}=0.12\right.$ for all the units except unit 1$)$ and $C_{F 2}\left(\beta=1.0, C_{F}=0.22\right)$. Figure 14 shows the horizontal profiles of macroscopic quantities in both microscopic and macroscopic simulation results. All these figures confirm that the difference in spatially-averaged mean velocity and turbulence in the microscopic simulations using two different grids is always small. In Fig. 14a to c, in contrast to the microscopic simulation results, macroscopic simulations using $C_{F B}$ (the coarse grid) have improved prediction in the velocity reduction profile than the other three Forchheimer coefficients at $z / H=1,0.5$ and $1 / 6 . C_{F A}$ and $C_{F 1}$ tend to over-predict the macroscopic streamwise velocity because $C_{F A}$ and $C_{F 1}$ (i.e. 0.12 ) are smaller than $C_{F B}$ and $C_{F 2}$ (i.e. 0.22). The constant Forchheimer coefficients $C_{F 2}$ (i.e. 0.22) evaluate the macroscopic streamwise velocity well at $z / H=0.5$, but under-predict it at the top interface $(z / H=1)$ and overestimate it near the ground $(z / H=1 / 6)$. Figure $14 d$ shows that all Forchheimer coefficients may predict the basic variation profile of the macroscopic vertical velocity, including the upward flow (positive value) near the windward edge, the weak vertical flow (i.e. nearly zero) in places far from the two ends, and the downward flow near the leeward edge of the porous region. For horizontal profiles of macroscopic TKE in Fig. 14e and $\mathrm{f}$, all macroscopic simulations overestimate the turbulence near the top interface $(z=H)$, and under-predict the turbulence near the windward edge of the porous region (near the location of $x / H=0$ ), but predict the shape of profiles well in places far from the two ends. Considering that microscopic simulations underestimate turbulence below the top interface (i.e. roof level $z=H$ ), in contrast to wind-tunnel data, it is difficult to estimate the prediction of turbulence using macroscopic simulations. Largeeddy simulations (LES) will be required to evaluate the accuracy of turbulence prediction in future work. Overall, a good sectional Forchheimer coefficients profile should be similar to $C_{F B}$ (see Fig. 13f), which is about 0.2 (a constant near to $C_{F 2}$ as $\beta=1$ ) at a medium height in the porous region, a little smaller than 0.2 near the top interface and much larger than 0.2 near the ground. Macroscopic simulation is a promising method for predicting the macroscopic velocity reduction through a porous region of urban canopy layers. 

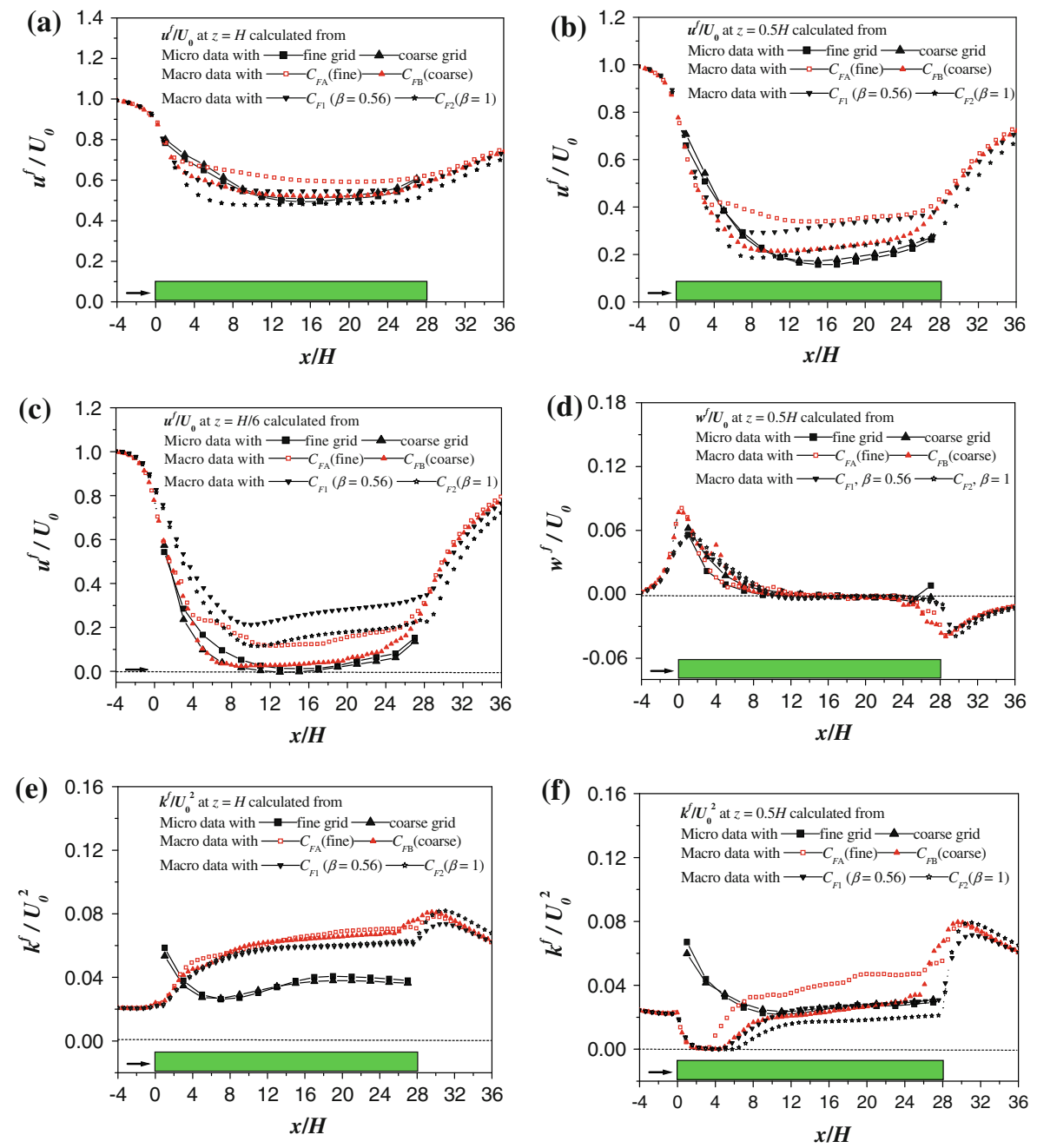

Fig. 14 Comparison of macroscopic properties between microscopic and macroscopic simulation results for the fourteen-row cube array. Macroscopic streamwise velocity at $\mathbf{a} z / H=1, \mathbf{b} z / H=0.5$, and $\mathbf{c} z / H=1 / 6$. d Macroscopic vertical velocity at $z / H=0.5$. Macroscopic TKE at e $z / H=1$, and $\mathbf{f} z / H=0.5$. For sectional spatially averaged values and Forchheimer coefficient $C_{F}(z)$ calculated in microscopic simulations, fine grid $\left(853 \times 23 \times 60 ; C_{F A}\right)$ and coarse grid $\left(583 \times 23 \times 47 ; C_{F B}\right)$ were used. For macroscopic simulations, we also used the Ergun equation $\left(C_{F 2}=0.22, \beta=1.0\right)$ and its revised equation $\left(C_{F 1}=0.12\right.$ as $\beta=0.56$ for all units except unit 1). $x=0$ is the windward edge of the porous region. $U_{0}(z)$ is the streamwise velocity in the free flow far upstream

\section{Conclusions}

We performed both microscopic simulations with a RANS $k-\varepsilon$ turbulence model and macroscopic simulations using a porous turbulence model for an investigation of urban turbulent flows through aligned cube arrays of 7, 14 and 21 rows, in which the aspect ratio is 1.0 and the porosity is 0.75 . Microscopic simulations were validated well by previous wind-tunnel data. We analysed 3D turbulent flow patterns in such cube arrays where the skimming flow 
regime exists in the secondary streets (canyon units) and channel flows are found in the main street (channel units), which is parallel to the approaching flow. There are both downward and upward flows across canyon roofs, together with both lateral inflow and outflow across the interface between canyons and their neighbouring channel units. The downward inflow rate across canyon roofs is a little larger than the upward outflow rate across canyon roofs, showing that the ventilation in $3 \mathrm{D}$ canyon flows differs from that in $2 \mathrm{D}$ canyon flows. The normalized flow rates and $\mathrm{ACHs}$ for canyons and channels were also estimated, for which we found the ventilation in channels is much better than that in canyons, and the air exchange for canyons due to turbulent fluctuations is more important than that due to the mean flow. We also analysed the microscopic variation of the streamwise velocity and vertical velocity, and the macroscopic velocity reduction through these cube arrays. Macroscopic quantities may be approximately fully developed when the cube array is sufficiently long, but the microscopic variables vary in a wave shape.

Then we carried out macroscopic simulations on porous media of these cube arrays using a macroscopic $k-\varepsilon$ turbulence model, with the sectional Forchheimer coefficients that were calculated from the microscopic results of the fine and coarse grids or the constant coefficients using Ergun equations. A single domain approach was used to deal with the interface conditions. The comparison between macroscopic and microscopic simulation results confirms that macroscopic simulations may predict the profile of velocity reduction well if a suitable profile of the Forchheimer coefficient is selected, but will under-predict the turbulence intensity near the windward edge of the porous region and over-predict the turbulence intensity near the top interface. The macroscopic fully developed characteristic appears if the porous region is sufficiently long. In the present porous turbulence model, the Darcy and Forchheimer terms mainly act as sink terms for the transport of momentum, TKE and its dissipation rate in most situations, and the magnitudes of Darcy terms are always much less than those of the Forchheimer terms. Overall, the present porous turbulence model with a series of analysis techniques provides a promising tool for numerical prediction of macroscopic wind conditions in porous cities with a large number of buildings.

Acknowledgements The study is supported by a grant from the Research Grants Council of the Hong Kong SAR Government (Project No. HKU 7145/07E).

Open Access This article is distributed under the terms of the Creative Commons Attribution Noncommercial License which permits any noncommercial use, distribution, and reproduction in any medium, provided the original author(s) and source are credited.

\section{References}

Antohe BV, Lage JL (1997) A general two-equation macroscopic turbulence model for incompressible flow in porous media. Int J Heat Mass Transf 40(13):3013-3024

Baik JJ, Kim JJ (2002) On the escape of pollutants from urban street canyons. Atmos Environ 36(3):527-536 Belcher SE (2005) Mixing and transport in urban areas. Philos Trans R Soc A 363:2947-2968

Belcher SE, Jerram N, Hunt JCR (2003) Adjustment of turbulent boundary layer to a canopy of roughness elements. J Fluid Mech 488:369-398

Britter RE, Hanna SR (2003) Flow and dispersion in urban areas. Annu Rev Fluid Mech 35:469-496

Brown MJ, Lawson RE, Decroix DS, Lee RL (2001) Comparison of centerline velocity measurements obtained around 2D and 3D buildings arrays in a wind tunnel. Report LA-UR-01-4138, Los Alamos National Laboratory, Los Alamos, 7 pp

Chan HC, Huang WC, Leu JM, Lai CJ (2007) Macroscopic modelling of turbulent flow over a porous medium. Int J Heat Fluid Flow 28(5):1157-1166 
Chandesris M, Serre G, Sagaut P (2006) A macroscopic turbulence model for flow in porous media suited for channel, pipe and rod bundle flows. Int J Heat Mass Transf 49(15-16):2739-2750

Chang CH, Meroney RN (2003) The effect of surroundings with different separation distances on surface pressures on low-rise buildings. J Wind Eng Ind Aerodyn 91(8):1039-1050

Cheng Y, Lien FS, Yee E, Sinclair R (2003) A comparison of large Eddy simulations with a standard $k-\varepsilon$ Reynolds-averaged Navier-Stokes model for the prediction of a fully developed turbulent flow over a matrix of cubes. J Wind Eng Ind Aerodyn 91(11):1301-1328

Choi CY, Waller PM (1997) Momentum transport mechanism for water flow over porous media. J Environ Eng-ASCE 123(8):792-799

Coceal O, Belcher SE (2005) Mean winds through an inhomogeneous urban canopy. Boundary-Layer Meteorol 115(1):47-68

de Lemos MJS (2005) Turbulent kinetic energy distribution across the interface between a porous medium and a clear region. Int Commun Heat Mass Transf 32(1-2):107-115

Ding A, Wang T, Zhao M, Wang TJ, Li ZK (2004) Simulation of sea-land breezes and a discussion of their implications on the transport of air pollution during a multi-day ozone episode in the Pearl River Delta of China. Atmos Environ 38(39):6737-6750

Etheridge D, Sandberg M (1996) Building ventilation: theory and measurement. Wiley, New York, 724 pp

Fenger J (1999) Urban air quality. Atmos Environ 33(29):4877-4900

Finnigan JJ (2000) Turbulence in plant canopies. Annu Rev Fluid Mech 32:519-571

Getachew D, Minkowycz WJ, Lage JL (2000) A modified form of the $k-\varepsilon$ model for turbulent flows of an incompressible fluid in porous media. Int J Heat Mass Transf 43(16):2909-2915

Hang J, Sandberg M, Li YG (2009) Effect of urban morphology on wind condition in idealized city models. Atmos Environ 43(4):869-878

Hanna SR, Tehranian S, Carissimo B, Macdonald RW, Lohner R (2002) Comparisons of model simulations with observations of mean flow and turbulence within simple obstacle. Atmos Environ 36(32):5067-5079

Kanda M (2006) Large-eddy simulations on the effects of surface geometry of building arrays on turbulent organized structures. Boundary-Layer Meteorol 118(1):151-168

Kuwahara F, Yamane I, Nakayama A (2006) Large eddy simulation of turbulent flow in porous media. Int Commun Heat Mass Transf 33(4):411-418

Li Y, Stathopoulos T (1997) Numerical evaluation of wind-induced dispersion of pollutants around a building. J Wind Eng Ind Aerodyn 67:757-766

Li XX, Liu CH, Leung DYC (2009) Numerical investigation of pollutant transport characteristics inside deep urban street canyons. Atmos Environ 43:2410-2418

Lien FS, Yee E (2004) Numerical modelling of the turbulent flow developing within and over a 3-D building array, part I: a high-resolution Reynolds-averaged Navier-Stokes approach. Boundary-Layer Meteorol 112(3):427-466

Lien FS, Yee E (2005) Numerical modelling of the turbulent flow developing within and over a 3-D building array, part III: a distributed drag force approach, its implementation and application. Boundary-Layer Meteorol 14(2):287-313

Lien FS, Yee E, Wilson JD (2005) Numerical modelling of the turbulent flow developing within and over a 3-d building array, part II: mathematical formulation for a distributed drag force approach. Boundary-Layer Meteorol 114(2):245-285

Liu HP, Zhang BY, Sang JG, Cheng AYS (2001) A laboratory simulation of plume dispersion in stratified atmospheres over complex terrain. J Wind Eng Ind Aerodyn 89(1):1-15

Liu CH, Leung DYC, Barth MC (2005) On the prediction of air and pollutant exchange rates in street canyons of different aspect ratios using large-eddy simulation. Atmos Environ 39(9):1567-1574

Martilli A, Santiago JL (2007) CFD simulation of airflow over a regular array of cubes. Part II: analysis of spatial average properties. Boundary-Layer Meteorol 122(3):635-654

Meroney RN, Pavegeau M, Rafailidis S, Schatzmann M (1996) Study of line source characteristics for 2-D physical modelling of pollutant dispersion in street canyons. J Wind Eng Ind Aerodyn 62(1):37-56

Nakayama A, Kuwahara F (1999) A macroscopic turbulence model for flow in a porous medium. J Fluid Eng-T ASME 121(2):427-433

Neale G, Nader W (1974) Practical significance of Brinkman's extension of Darcy's law: coupled parallel flows within a channel and a bounding porous medium. Can J Chem Eng 52(4):475-478

Oke TR (1988) Street design and urban canopy layer climate. Energy Build 11(1-3):103-113

Pedras MHJ, de Lemos MJS (2000) On the definition of turbulent kinetic energy for flow in porous media. Int Commun Heat Mass Transf 27(2):211-220

Pedras MHJ, de Lemos MJS (2001) Macroscopic turbulence modelling for incompressible flow through undeformable porous media. Int J Heat Mass Transf 44(6):1081-1093 
Prakash M, Turan OF, Li YG, Mahoney J, Thorpe GR (2001a) Impinging round jet studies in a cylindrical enclosure with and without a porous layer: part I-flow visualizations and simulations. Chem Eng Sci 56(12):3855-3878

Prakash M, Turan OF, Li YG, Mahoney J, Thorpe GR (2001b) Impinging round jet studies in a cylindrical enclosure with and without a porous layer: part II-LDV measurements and simulations. Chem Eng Sci 56(12):3879-3892

Santiago JL, Martilli A, Martin F (2007) CFD simulation of airflow over a regular array of cubes. Part I: Three-dimensional simulation of the flow and validation with wind-tunnel measurements. BoundaryLayer Meteorol 122(3):609-634

Santiago JL, Coceal O, Martilli A, Belcher SE (2008) Variation of the sectional drag coefficient of a group of buildings with packing density. Boundary-Layer Meteorol 128(3):445-457

Silva RA, de Lemos MJS (2003) Turbulent flow in a channel occupied by a porous layer considering the stress jump at the interface. Int J Heat Mass Transf 46(26):5113-5121

Sini JF, Anquetin S, Mestayer PG (1996) Pollutant dispersion and thermal effects in urban street canyons. Atmos Environ 30(15):2659-2677

Tong H, Walton A, Sang JG, Chan JCL (2005) Numerical simulation of the urban boundary layer over the complex terrain of Hong Kong. Atmos Environ 39(19):3549-3563

Xie XM, Huang Z, Wang J (2006) The impact of urban street layout on local atmospheric environment. Build Environ 41(10):1352-1363 